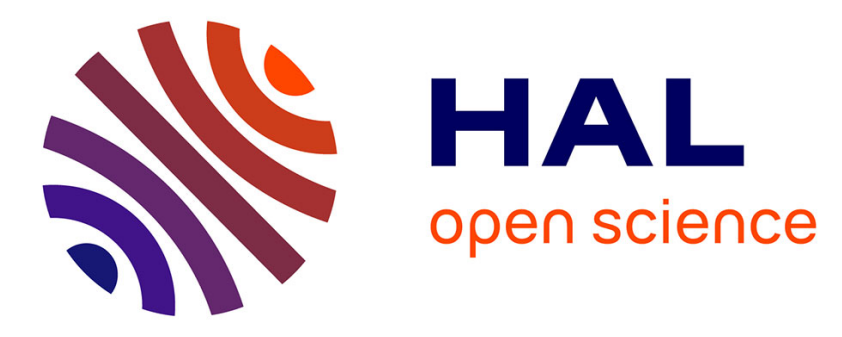

\title{
A Fast Fourier Transform-based approach for Generalized Disclination Mechanics within a Couple Stress theory
}

Stéphane Berbenni, Vincent Taupin, Claude Fressengeas, Laurent Capolungo

\section{- To cite this version:}

Stéphane Berbenni, Vincent Taupin, Claude Fressengeas, Laurent Capolungo. A Fast Fourier Transform-based approach for Generalized Disclination Mechanics within a Couple Stress theory. Holm Altenbach; Samuel Forest. Generalized Continua as Models for Classical and Advanced Materials, Springer, pp.47-75, 2016, 978-3-319-31719-9. hal-02360511

\section{HAL Id: hal-02360511 \\ https://hal.science/hal-02360511}

Submitted on 12 Nov 2019

HAL is a multi-disciplinary open access archive for the deposit and dissemination of scientific research documents, whether they are published or not. The documents may come from teaching and research institutions in France or abroad, or from public or private research centers.
L'archive ouverte pluridisciplinaire HAL, est destinée au dépôt et à la diffusion de documents scientifiques de niveau recherche, publiés ou non, émanant des établissements d'enseignement et de recherche français ou étrangers, des laboratoires publics ou privés. 


\title{
A Fast Fourier Transform-based approach for Generalized Disclination Mechanics within a Couple Stress theory
}

\author{
Stéphane Berbenni and Vincent Taupin and Claude Fressengeas and Laurent Capolungo
}

\begin{abstract}
Recently, a small-distortion theory of coupled plasticity and phase transformation accounting for the kinematics and thermodynamics of generalized defects called generalized disclinations (abbreviated gdisclinations) has been proposed [2,3]. Then, a first numerical spectral approach has been developed to solve the elasto-static equations of field dislocation and g-disclination mechanics set out in this theory for periodic media and for linear elastic media using the classic Hooke's law [6]. Here, given a spatial distribution of generalized disclination density tensors in a homogenous linear higher order elastic media described, a couple stress theory with elastic incompatibilities of first and second orders is developed. The incompatible and compatible elastic second and first distortions are obtained from the solution of Poisson and Navier-type equations in the Fourier space. The efficient Fast Fourier Transform (FFT) algorithm [17] is used based on intrinsic Discrete Fourier Transforms (DFT) that are well adapted to the discrete grid to compute higher order partial derivatives in the Fourier space. Therefore, stress and couple stress fields can be calculated using the inverse FFT. The numerical examples are given for straight wedge disclinations and associated wedge disclination dipoles which are of importance to geometrically describe tilt grain boundaries at fine scales in polycrystalline solids.
\end{abstract}

\section{Introduction}

In crystalline media, the internal stresses and couple stresses result from an incompatible process where crystal defects - dislocations, disclinations or "generalized disclinations" (abbreviated "g-disclinations") - induce the discontinuity of (elastic, i.e. lattice) displacement or distortion across surfaces in the body. Dislocations and disclinations were mathematically introduced by Volterra [54]. In the sole presence of dislocations, incompatibility fields were smoothly described in the continuum theory of dislocations initiated by Kröner [26, 29] and many others [7, 38, 55, 25] by using Nye's dislocation density tensor [43]. The continuum theory of dislocations was recently revisited by Acharya and co-workers [1, 47, 4]. One of the key features of the revisited continuum dislocation theory resides in the Stokes-Helmholtz decomposition of the elastic distortion and the associated side conditions yielding a unique solution for the incompatible part associated with a prescribed dislocation density field, while the compatible part is unambiguously determined

Stéphane Berbenni

Laboratoire d'Etude des Microstructures et de Mécanique des Matériaux, LEM3, UMR CNRS 7239, University of Lorraine, Ile du Saulcy, 57045 Metz, France e-mail: stephane.berbenni@univ-lorraine.fr

Vincent Taupin

Laboratoire d'Etude des Microstructures et de Mécanique des Matériaux, LEM3, UMR CNRS 7239, University of Lorraine, Ile du Saulcy, 57045 Metz, France e-mail: vincent.taupin@univ-lorraine.fr

Claude Fressengeas

Laboratoire d'Etude des Microstructures et de Mécanique des Matériaux, LEM3, UMR CNRS 7239, University of Lorraine, Ile du Saulcy, 57045 Metz, France e-mail: claude.fressengeas@univ-lorraine.fr

Laurent Capolungo

George Woodruff School of Mechanical Engineering, Georgia Institute of Technology, UMI 2958 Georgia Tech-CNRS, 57070

Metz, France e-mail: laurent.capolungo@me.gatech.edu 
from the satisfaction of the balance of linear momentum together with boundary conditions. When disclinations are present in the body in addition to dislocations, the displacement and rotation vectors are both multi-valued functions. Such a situation typically occurs in solids exhibiting kink bands, grain and subgrain boundaries and triple junctions. In this case, the elastic curvature tensor has an incompatible part complementing the compatible gradient component $[9,15]$. Beyond Volterra's construct, the entire distortion tensor including the strain tensor in addition to the rotation tensor may be multivalued along some surface. Such situations are commonplace in materials science. They include terminating twinning and phase boundaries, terminating shear bands, sharp corners of inclusions in a matrix of dissimilar media, in addition to grain boundaries and triple junctions. As recently discussed in [2], the discontinuity of the distortion field is reflected by the incompatibility of the elastic 2-distortion, (i.e. the second gradient of displacement in strain gradient elasticity theory) in the presence of a non-vanishing g-disclination density tensor field. A Weingarten theorem and a finite strain framework were also recently reported in [3].

An increasingly attractive alternative to the finite-element method is a computationally efficient scheme based on the Fast Fourier Transform (FFT) for the solution of periodic boundary-value problems in continuum mechanics. Pioneering works in this field can be found in [35, 37, 36, 11, 13, 30, 33, 41, 53]. This numerical approach solves the Lippmann-Schwinger integral equation of the periodic boundary-value problems by means of the Green's function of a chosen reference medium. It has been applied so far to elastic and elasto-plastic composites and polycrystals in the absence of crystal defects. The main interest of the FFT approach relies on its computational efficiency [36, 44]. Its main drawbacks are the need for a periodic representative volume element and the possible occurrence of spurious Gibbs oscillations arising from the presence of strong spatial gradients. The elasto-static equations of FDM, which provide the long-range internal elastic fields associated with a prescribed distribution of dislocation densities in a body, were recently solved within the FFT framework [8]. In the latter, the equations for the incompatible elastic distortions and the balance of momentum are solved in the Fourier space, while the resulting elastic fields are obtained in the real space by using the inverse Fourier transforms. Independently, an extension of this spectral approach to field dislocation and generalized-disclination mechanics (FDGDM) was first proposed in [6] using classic Cauchy stress theory, with additional features including a different discretization treatment of FFT-induced Gibbs oscillations in comparison with ref. [8]. Extensive 2D simulations showed that the numerical spectral approach is as accurate as optimized finite element approximations, but computationally much more efficient [6].

Motivated by the accuracy and the speed of such spectral approaches for the solution of classic elasto-static problems, we extend in the present contribution the theory developed in [6] to account for the second order couple stress tensor (which is related to the skew-symmetric part of the third order hyperstress tensor) and the second order elastic curvature (which is related to the third order elastic 2-distortion tensor). Among various higher order theories, the Cosserat, couple-stress, micromorphic, strain-gradient theories are mostly documented, see e.g. [28], [42], [12] and [14]. The couple-stress theory originally developed by [34, 24] contains the least material parameters in the constitutive equations compared with other non-conventional theories involving multiple materials length scale dependent elastic constants which may be difficult to identify at fine scales. Analytical elastic fields of straight dislocations and disclinations were obtained in a couple stress theory in $[27,32,5,19]$. Furthermore, in a different context dedicated to homogenization and composites, the Green's function technique for isotropic centrosymmetric couple stress materials was derived in $[48,56]$ and a DFT-based approach was proposed for both Cosserat and couple stress linear elastic heterogeneous materials in [22, 23]. Recently, a general free energy density functional for crystalline materials with third order hyperstress tensor undergoing incompatible fields due to dislocations, disclinations and g-disclinations was proposed in [52,50]. Here, the constitutive model will be built up starting from this general free energy density functional but will be simplified to only consider the second order deviatoric elastic curvature within a couple stress theory with incompatibilities.

The paper is organized as follows:

In section 2, the notations are introduced. The kinematics for generalized disclination (abbreviated gdisclination in the sequel) mechanics is reviewed in section 3 and the solutions for incompatible fields are given in the same section. Then, the generalized constitutive and equilibrium equations within a couple stress theory are introduced in section 4 . General three-dimensional solutions for incompatible elastic fields of g-disclinations are derived in section 5 to compute their stress and couple stress fields in the Fourier 
space. In section 6, the DFT method is introduced in the case of two-dimensional (2D) problems, and the FFT algorithm [17] will be used to solve the Poisson and Navier-type equations with microstructural length scale in the case of infinite straight g-disclinations. In section 7, g-disclination densities are distributed on 2D FFT pixelized grids for different configurations: single wedge disclination and wedge disclination dipole. The incompatible and compatible elastic fields are obtained in the discrete Fourier space and then used to derive the stresses and couple stresses by using the inverse FFT for an isotropic centrosymmetric elastic solid. The present numerical method is validated by comparisons with existing analytical expressions $[10,5,46]$.

\section{Notations}

A bold symbol denotes a tensor or a vector. The symmetric part of tensor $\mathbf{A}$ is denoted $\mathbf{A}^{\text {sym }}$. Its skewsymmetric part is $\mathbf{A}^{\text {skew }}$ and its transpose is denoted by $\mathbf{A}^{t}$. The tensor $\mathbf{A} \cdot \mathbf{B}$, with rectangular Cartesian components $A_{i k} B_{k j}$, results from the dot product of tensors $\mathbf{A}$ and $\mathbf{B}$, and $\mathbf{A} \otimes \mathbf{B}$ is their tensorial product, with components $A_{i j} B_{k l}$. The vector $\mathbf{A} \cdot \mathbf{V}$, with rectangular Cartesian components $A_{i j} V_{j}$, results from the dot product of tensor $\mathbf{A}$ and vector $\mathbf{V}$. A ":" represents the trace inner product of the two second order tensors $\mathbf{A}: \mathbf{B}=A_{i j} B_{i j}$, in rectangular Cartesian components, or the product of a higher order tensor with a second order tensor, e.g., $\mathbf{A}: \mathbf{B}=A_{i j k l} B_{k l}$. A “:” represents the trace inner product of the two third order tensors $\mathbf{A}: \mathbf{B}=A_{i j k} B_{i j k}$, in rectangular Cartesian components, or, it denotes the product of a higher order tensor with a third order tensor, e.g., $\mathbf{A} \vdots \mathbf{B}=A_{i j k l m} B_{k l m}$. The cross product of a second-order tensor $\mathbf{A}$ and a vector $\mathbf{V}$, the div and curl operations for second/third-order tensors are defined row by row, in analogy with the vectorial case. For any base vector $\mathbf{e}_{i}$ of the reference frame:

$$
\begin{aligned}
(\mathbf{A} \times \mathbf{V})^{t} \cdot \mathbf{e}_{i} & =\left(\mathbf{A}^{t} \cdot \mathbf{e}_{i}\right) \times \mathbf{V} \\
(\operatorname{div} \mathbf{A})^{t} \cdot \mathbf{e}_{i} & =\operatorname{div}\left(\mathbf{A}^{t} \cdot \mathbf{e}_{i}\right) \\
(\operatorname{curl} \mathbf{A})^{t} \cdot \mathbf{e}_{i} & =\operatorname{curl}\left(\mathbf{A}^{t} \cdot \mathbf{e}_{i}\right) .
\end{aligned}
$$

In rectangular Cartesian components:

$$
\begin{aligned}
(\mathbf{A} \times \mathbf{V})_{i j} & =e_{j k l} A_{i k} V_{l} \\
(\mathbf{A} \times \mathbf{V})_{i j k} & =e_{k l m} A_{i j l} V_{m} \\
(\operatorname{divA})_{i} & =A_{i j, j} \\
(\operatorname{divA})_{i j} & =A_{i j k, k} \\
(\operatorname{curl} \mathbf{A})_{i j} & =e_{j k l} A_{i l, k}=-(\operatorname{grad} \mathbf{A}: \mathbf{X})_{i j} \\
(\operatorname{curl} \mathbf{A})_{i j k} & =e_{k l m} A_{i j m, l} .
\end{aligned}
$$

where $e_{j k l}$ is a component of the third-order alternating Levi-Civita tensor $\mathbf{X}$ and the spatial derivative with respect to a Cartesian coordinate is indicated by a comma followed by the component index.

\section{Kinematics of generalized crystal defects and incompatibilities}

\subsection{Linear theory}

The analysis is developed in the small distortion framework (linear theory). The body $V$, with boundary $\partial V$, is assumed to be a continuum, with smooth displacement and rotation vector fields $(\mathbf{u}, \boldsymbol{\omega}=1 / 2$ curlu $)$. The total 1-distortion (first distortion) tensor field $\mathbf{U}=\operatorname{grad} \mathbf{u}$, the curvature tensor field, $\boldsymbol{\kappa}=\operatorname{grad} \boldsymbol{\omega}$, and the 2distortion (second distortion) tensor field, $\mathbf{G}=\operatorname{grad} \mathbf{U}$, are therefore assumed to be integrable (compatible, or curl free). Under such assumptions, the possibility of developing cracks in the body is discarded. The 
total 1-distortion writes as the sum of the elastic distortion, $\mathbf{U}_{e}$, and plastic distortion, $\mathbf{U}_{p}$ :

$$
\mathbf{U}=\mathbf{U}_{e}+\mathbf{U}_{p}
$$

Similarly, the 2-distortion tensor can be decomposed into elastic and plastic 2-distortion tensors:

$$
\mathbf{G}=\mathbf{G}_{e}+\mathbf{G}_{p}
$$

In a compatible body in the absence of polarized crystal defect density, the elastic/plastic distortions and 2-distortions are curl-free gradient tensors. However, they will contain incompatible, non-gradient parts, in the presence of a polarized crystal defect density, while total 1 and 2-distortions remain compatible. Such general incompatibilities are now discussed in terms of crystal defects.

\subsection{Volterra's crystal translation and rotation line defects}

Volterra [54] introduced six types of crystal line defects. Three of them, known as dislocations, are translational defects, and the other three, referred to as disclinations, are rotational defects. Like disclinations, dislocations have a smooth elastic distortion field $\mathbf{U}_{e}$ in a non-simply-connected domain excluding their core. However, their (elastic) displacement field features a discontinuity denoted $\llbracket \mathbf{u}_{e} \rrbracket$ across a (non-unique) smooth surface in this domain. The geometry of any such surface is arbitrary except that, in a discrete modeling framework, it terminates along the dislocation line. A line integral of the elastic distortion field along any curve encircling the dislocation line, i.e. a Burgers circuit, is constant and is equal to the discontinuity of the elastic displacement. This constant $\mathbf{b}=\llbracket \mathbf{u}_{e} \rrbracket$ is referred to as the Burgers vector of the dislocation. It represents the strength of the dislocation. In contrast with Volterra's discrete representation of crystal defects, we presently choose a continuous setting, in order to regularize this classical description. We consider smooth elastic distortion fields in simply connected domains, in which they are point-wise irrotational outside the core region, whereas their non-vanishing curl defines a smooth dislocation density tensor field inside the core (of non-zero volume):

$$
\boldsymbol{\alpha}=\operatorname{curl} \mathbf{U}_{e} .
$$

The Burgers vector is then obtained by integrating the dislocation density tensor field, referred to as Nye's tensor field, along appropriate surface patches $S$ with unit normal $\mathbf{n}$ :

$$
\mathbf{b}=\int_{S} \boldsymbol{\alpha} \cdot \mathbf{n} d S
$$

Similarly, disclinations result from a discontinuity denoted $\llbracket \boldsymbol{\omega}_{e} \rrbracket$ in the rotation field over a surface terminating on the disclination line in a discrete setting, even though a smooth elastic curvature field $\boldsymbol{\kappa}_{e}$ exists in this region. The strength of disclinations is characterized by their Frank vector $\boldsymbol{\Omega}$, which represents the magnitude and direction of the rotational discontinuity $\boldsymbol{\Omega}=\llbracket \boldsymbol{\omega}_{e} \rrbracket$ over a closed circuit encircling the disclination line. In deWit's continuous setting [9], also adopted in the present paper, the smooth elastic curvature field is irrotational outside the disclination core region, and the disclination density tensor is defined as the curl of this field inside the core, of non-zero volume:

$$
\boldsymbol{\theta}=\operatorname{curl} \boldsymbol{\kappa}_{e} .
$$

The Frank vector is then obtained by integrating the disclination density tensor field along appropriate surface patches $S$ :

$$
\boldsymbol{\Omega}=\int_{S} \boldsymbol{\theta} . \mathbf{n} d S
$$




\subsection{Generalized disclination (g-disclination) kinematics}

Acharya and Fressengeas [2] adopted a similar approach in introducing generalized disclinations , as shown in Fig. 1. The g-disclination concept goes beyond the Volterra construct, in the sense that the distortion field now has a discontinuity denoted $\llbracket \mathbf{U}_{e} \rrbracket$ along a surface terminating at the g-disclination line (Fig. 1), whereas the elastic 2-distortion tensor field $\mathbf{G}_{e}$ is still smooth in the non-simply connected region excluding the g-disclination line. As already mentioned, this surface of discontinuity is referred to as a phase or grain boundary. The strength $\Pi$ of the g-disclination is defined as the jump in the elastic distortion tensor field across the interphase: $\boldsymbol{\Pi}=\llbracket \mathbf{U}_{e} \rrbracket$. In a continuous setting, the elastic 2-distortion field is point-wise irrotational in the defect free volume of the body. Its curl in the defected part provides for the definition of the third order g-disclination density tensor field $\pi$ :

$$
\boldsymbol{\pi}=\operatorname{curlG} \mathbf{G}_{e}
$$

and the integration of the latter over an appropriate surface patch yields the jump of the elastic distortion tensor field:

$$
\Pi=\int_{S} \boldsymbol{\pi} \cdot \mathbf{n} d S
$$

When, as a special case, the strain discontinuity vanishes, while a rotation discontinuity $\llbracket \boldsymbol{\omega}_{e} \rrbracket$ is persisting, the g-disclinations reduce to standard disclinations. In the context of g-disclinations, the dislocation density tensor $\boldsymbol{\alpha}$ needs to be redefined by alternating the elastic 2 -distortion tensor [2]:

$$
\boldsymbol{\alpha}=-\mathbf{G}_{e}: \mathbf{X}
$$

instead of Eq.12.

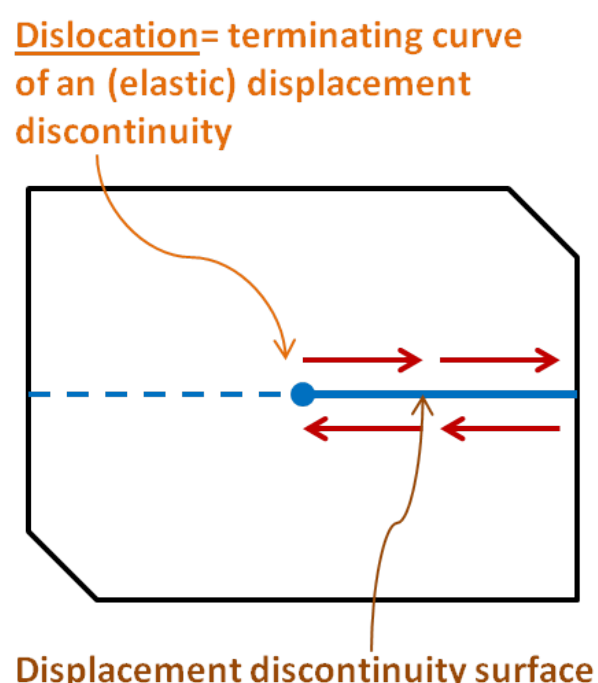

(a)

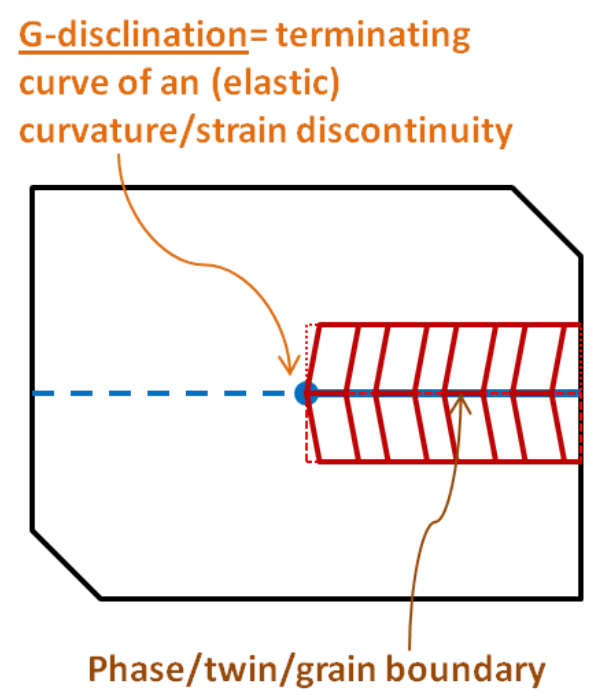

(b)

Fig. 1 Cross sectional view of two different types of straight line defects: dislocation (a) seen as the terminating curve of the surface of displacement discontinuity (the arrows with reverse directions along the displacement discontinuity surface describe different displacement directions), g-disclination (b) seen as the terminating curve of the surface of curvature/strain discontinuity (the differently inclined parallel lines in the vicinity of the distortion discontinuity surface decribe different strains like different shears for example). 


\subsection{Incompatible field equations}

Invoking the Stokes-Helmholtz orthogonal decomposition of the square-integrable elastic 2-distortion tensor field $\mathbf{G}_{e}$ (see for example [21]), there exist unique tensor fields $\boldsymbol{\chi}$ and $\mathbf{Z}$ such that $\mathbf{G}_{e}$ writes as the sum:

$$
\mathbf{G}_{e}=\mathbf{G}_{e}^{\perp}+\mathbf{G}_{e}^{\|}=\operatorname{curl} \boldsymbol{\chi}+\operatorname{grad} \mathbf{Z} .
$$

with the orthogonality condition $\int_{V} \operatorname{curl} \chi: \operatorname{grad} \mathbf{Z} d v=0$. Thus, taking the curl of $\mathbf{G}_{e}$ in Eq.19 extracts $\operatorname{curl} \boldsymbol{\chi}$ and discards $\operatorname{grad} \mathbf{Z}$, whereas taking its divergence extracts $\operatorname{grad} \mathbf{Z}$ and eliminates $\operatorname{curl} \boldsymbol{\chi}$. Therefore, Eq.16 involves only $\operatorname{curl} \boldsymbol{\chi}$, which we will identify below as the incompatible part $\mathbf{G}_{e}^{\perp}$ of $\mathbf{G}_{e}$ :

$$
\operatorname{curlG}{ }_{e}^{\perp}=\operatorname{curl} \operatorname{curl} \chi=\pi .
$$

Similarly, $\operatorname{grad} \mathbf{Z}$ will be the compatible part $\mathbf{G}_{e}^{\|}$of the elastic 2-distortion $\mathbf{G}_{e}$, and $\mathbf{Z}$ will be the elastic distortion $\mathbf{U}_{e}$, up to a constant. To ensure correctness of this identification, $\mathbf{G}_{e}^{\perp}$ must vanish identically throughout the body when $\boldsymbol{\pi}=0$. In this aim, following [21, 1], Eq.20 is augmented with the side conditions:

$$
\begin{aligned}
\operatorname{div} \mathbf{G}_{e}^{\perp} & =0 \text { in } V \\
\mathbf{G}_{e}^{\perp} \cdot \mathbf{n} & =0 \text { on } \partial V
\end{aligned}
$$

with unit normal $\mathbf{n}$ on $\partial V$. Then taking the curl of Eq.16 and using the side condition (21), it follows that:

$$
\operatorname{curlcurl} \mathbf{G}_{e}^{\perp}=\operatorname{grad\operatorname {div}} \mathbf{G}_{e}^{\perp}-\operatorname{div} \operatorname{grad} \mathbf{G}_{e}^{\perp}=-\operatorname{div} \operatorname{grad} \mathbf{G}_{e}^{\perp}=\operatorname{curl} \boldsymbol{\pi}
$$

Hence, $\mathbf{G}_{e}^{\perp}$ satisfies a first Poisson-type equation

$$
\begin{aligned}
\operatorname{div} \operatorname{grad} \mathbf{G}_{e}^{\perp} & =-\operatorname{curl} \boldsymbol{\pi} \text { in } V \\
\mathbf{G}_{e}^{\perp} \cdot \mathbf{n} & =0 \text { on } \partial V .
\end{aligned}
$$

In component form, Eq.24 reads

$$
G_{i j k, l l}^{e, \perp}=-e_{k l m} \pi_{i j m, l}
$$

As a consequence, the field of incompatible elastic 2-distortion $\mathbf{G}_{e}^{\perp}$ is uniquely determined once the $\mathrm{g}$ disclination density field $\boldsymbol{\pi}$ is prescribed. In particular, it vanishes uniformly when $\boldsymbol{\pi}=0$. Using Eqs. $(8,18)$, the dislocation density tensor can therefore be written as:

$$
\boldsymbol{\alpha}=-\mathbf{G}_{e}^{\perp}: \mathbf{X}-\operatorname{grad} \mathbf{U}_{e}: \mathbf{X}=\operatorname{curl} \mathbf{U}_{e}-\mathbf{G}_{e}^{\perp}: \mathbf{X}
$$

In turn, the Stokes-Helmholtz decomposition of the elastic distortion $\mathbf{U}_{e}$ can be used to separate its compatible part, $\mathbf{U}_{e}^{\|}$, from its incompatible part, $\mathbf{U}_{e}^{\perp}$ :

$$
\mathbf{U}_{e}=\mathbf{U}_{e}^{\perp}+\mathbf{U}_{e}^{\|}=\operatorname{curl} \psi+\operatorname{grad} \mathbf{w}
$$

and to ensure uniqueness of the latter through the solution of a Poisson-type equation. In the decomposition (28), $\mathbf{U}_{e}^{\|}=$grad w again belongs to the null-space of the curl operator since curl grad $\mathbf{w}=0$, while $\mathbf{U}_{e}^{\perp}=$ curl $\psi$ must additionally satisfy the side conditions:

$$
\begin{aligned}
\operatorname{div} \mathbf{U}_{e}^{\perp} & =0 \text { in } V \\
\mathbf{U}_{e}^{\perp} \cdot \mathbf{n} & =0 \text { on } \partial V .
\end{aligned}
$$

Invoking the identity curl curl $\mathbf{U}_{e}^{\perp}=\operatorname{grad} \operatorname{div} \mathbf{U}_{e}^{\perp}-\operatorname{div} \operatorname{grad} \mathbf{U}_{e}^{\perp}$, taking the curl of Eq. 27 and using Eq.29 then leads to a second Poisson-type equation:

$$
\begin{aligned}
\operatorname{div} \operatorname{grad} \mathbf{U}_{e}^{\perp} & =-\operatorname{curl}\left(\boldsymbol{\alpha}+\mathbf{G}_{e}^{\perp}: \mathbf{X}\right) \text { in } V \\
\mathbf{U}_{e}^{\perp} \cdot \mathbf{n} & =0 \text { on } \partial V .
\end{aligned}
$$

In component form, Eq.31 reads 


$$
U_{i j, k k}^{e, \perp}=-e_{j k l} \alpha_{i l, k}-\left(G_{i j k}^{e, \perp}-G_{i k j}^{e, \perp}\right)_{, k}
$$

Hence, $\mathbf{U}_{e}^{\perp}$ is uniquely determined once the dislocation and g-disclination density fields $(\boldsymbol{\alpha}, \boldsymbol{\pi})$ are prescribed. In particular, it vanishes uniformly in $V$ when $\boldsymbol{\alpha}=0$ and $\boldsymbol{\pi}=0$. Eqs. $(31,33)$ will be transformed in the Fourier space in what follows (Section 5) and solved using the discrete Fourier transform (DFT) method (Section 6).

\section{Constitutive and equilibrium equations}

\subsection{Constitutive relationships}

Recently, a general free energy density functional $\Psi\left(\boldsymbol{\varepsilon}_{e}, \mathbf{G}_{e}\right)$ for crystalline materials undergoing incompatible fields due to dislocations, disclinations and g-disclinations was reported in [52]. Here, in order to reduce this general form to a couple stress theory with incompatible fields, $\Psi$ is supposed to only depend on the elastic strain $\boldsymbol{\varepsilon}_{e}$ and the skew-symmetric part of the elastic 2-distortion tensor $\mathbf{G}_{e}$ denoted $\mathbf{G}_{e}^{\text {skew }}$ as follows:

$$
\Psi=\frac{1}{2} \boldsymbol{\varepsilon}_{e}: \mathbf{C}: \boldsymbol{\varepsilon}_{e}+\boldsymbol{\varepsilon}_{e}: \mathbf{B}: \mathbf{G}_{e}^{\text {skew }}+\mathbf{G}_{e}^{\text {skew }}: \mathbf{D}: \boldsymbol{\varepsilon}_{e}+\frac{1}{2} \mathbf{G}_{e}^{\text {skew }}: \mathbf{E}: \mathbf{G}_{e}^{\text {skew }}
$$

where $\mathbf{C}$ is the fourth order tensor of linear elastic moduli with the classic symmetry properties $C_{i j k l}=$ $C_{j i k l}=C_{i j l k}=C_{k l i j}$, B is a fifth order tensor with $B_{i j k l m}=B_{j i k l m}=-B_{i j l k m}=-B_{j i l k m}$, D is a fifth order tensor with $D_{i j k l m}=-D_{j i k l m}=D_{i j k m l}=-D_{j i k m l}$ and $\mathbf{E}$ is a sixth order tensor with $E_{i j k l m n}=-E_{j i k l m n}=$ $-E_{i j k m l n}=E_{j i k m l n}=E_{l m n i j k}$. The constitutive relationships are obtained by taking the partial derivatives of $\boldsymbol{\Psi}$ with respect to $\boldsymbol{\varepsilon}_{e}$ and to $\mathbf{G}_{e}^{\text {skew }}$ in order to find the symmetric second order stress tensor $\mathbf{T}^{\text {sym }}$ (i.e. $T_{i j}^{s y m}=T_{j i}^{\text {sym }}$ ) and the skew-symmetric third order hyperstress tensor denoted $\mathbf{M}^{\text {skew }}$ (i.e. $M_{i j k}^{\text {skew }}=-M_{j i k}^{\text {skew }}$ ), respectively:

$$
\begin{aligned}
\mathbf{T}^{\text {sym }} & =\mathbf{C}: \boldsymbol{\varepsilon}_{e}+\mathbf{B}: \mathbf{G}_{e}^{\text {skew }}+\mathbf{G}_{e}^{\text {skew }}: \mathbf{D} \\
\mathbf{M}^{\text {skew }} & =\boldsymbol{\varepsilon}_{e}: \mathbf{B}+\mathbf{D}: \boldsymbol{\varepsilon}_{e}+\mathbf{E}: \mathbf{G}_{e}^{\text {skew }}
\end{aligned}
$$

For isotropic and centrosymmetric materials as considered here, the free energy density functional $\Psi$ further reduces to the one of a couple stress material $[34,24]$ with elastic incompatibilities under the quadratic form:

$$
\Psi=\frac{1}{2} \boldsymbol{\varepsilon}_{e}: \mathbf{C}: \boldsymbol{\varepsilon}_{e}+\frac{1}{2} \boldsymbol{\kappa}_{e}^{D}: \mathbf{A}: \boldsymbol{\kappa}_{e}^{D}
$$

where $\boldsymbol{\kappa}_{e}^{D}$ is the second order deviatoric elastic curvature tensor, and, $\mathbf{C}, \mathbf{A}$ read:

$$
\begin{aligned}
& C_{i j k l}=\lambda \delta_{i j} \delta_{k l}+\mu\left(\delta_{i k} \delta_{j l}+\delta_{j k} \delta_{i l}\right) \\
& A_{i j k l}=A_{1} \delta_{i k} \delta_{j l}-A_{2} \delta_{j k} \delta_{i l}
\end{aligned}
$$

where $\mu$ and $\lambda$ are respectively the classic shear modulus and Lamé constant of the material, and, $A_{1}, A_{2}$ are couple stress elastic constants that are length scale dependent. Let us note that the second order elastic curvature tensor $\boldsymbol{\kappa}_{e}$ is related to the skew-symmetric part of the third order elastic 2-distortion tensor $\mathbf{G}_{e}^{\text {skew }}$ as follows:

$$
\begin{aligned}
\boldsymbol{\kappa}_{e} & =-\frac{1}{2} \mathbf{X}: \mathbf{G}_{e}^{\text {skew }} \\
\mathbf{G}_{e}^{\text {skew }} & =-\mathbf{X} \cdot \boldsymbol{\kappa}_{e}
\end{aligned}
$$

Taking now the thermodynamic conjugate of $\boldsymbol{\kappa}_{e}^{D}$ as the second order deviatoric couple stress tensor $\mathbf{m}^{D}$, we obtain from equation (37) together with equation (38) the constitutive relationships for homogeneous isotropic centro-symmetric materials: 


$$
\begin{aligned}
T_{i j}^{s y m} & =C_{i j k l} \varepsilon_{k l}^{e}=\lambda \varepsilon_{k k}^{e} \delta_{i j}+2 \mu \varepsilon_{i j}^{e} \\
m_{i j}^{D} & =A_{i j k l} \kappa_{k l}^{e D}=A_{1} \kappa_{i j}^{e D}-A_{2} \kappa_{j i}^{e D}
\end{aligned}
$$

Let us note that only the deviatoric parts of second order elastic curvature and couple stress tensors are constitutively determined like in the so-called "undeterminate" couple stress theory originally derived by [34]. The second order couple stress tensor $\mathbf{m}$ is related to the skew-symmetric part of the third order hyperstress tensor $\mathbf{M}^{\text {skew }}$ by the following operations:

$$
\begin{aligned}
\mathbf{m} & =-\mathbf{X}: \mathbf{M}^{\text {skew }} \\
\mathbf{M}^{\text {skew }} & =-\frac{1}{2} \mathbf{X} \cdot \mathbf{m}
\end{aligned}
$$

This initial couple stress theory is still very controversial, see for instance [40], who proposed from homogenization theory with micro-randomness a couple stress theory with symmetric couple stress tensor. In the present study, the material constant $A_{1}$ is defined as a function of a microstructural length scale (or characteristic size) $l$ such that $A_{1}=4 \mu l^{2}$ [34]. Furthermore, the constant $A_{2}$ is bounded in the Mindlin-Tiersten theory [34].

\subsection{Equilibrium equations}

In a couple stress theory, the linear and angular momentum balance equations for the elasto-static problem without body force and body couple force densities take the forms:

$$
\begin{aligned}
\operatorname{div} \mathbf{T} & =0 \text { in } V, \\
\operatorname{div} \mathbf{m}^{D}-\mathbf{X}: \mathbf{T} & =0, \text { in } V
\end{aligned}
$$

where $\mathbf{T}$ is the force-stress tensor which splits into symmetric $\mathbf{T}^{\text {sym }}$ and skew-symmetric $\mathbf{T}^{\text {skew }}$ parts: $\mathbf{T}=$ $\mathbf{T}^{\text {sym }}+\mathbf{T}^{\text {skew }}$ and where the second order tensor $\mathbf{m}^{D}$ is the deviatoric couple-stress tensor as defined earlier.

The equilibrium equations (46) and (47) are appended with force-stress vector field $\mathbf{t}^{d}$ and couple-stress vector $\mathbf{m}^{d}$ applied to a part of the boundary $\partial V_{t}$ as detailed in [34] (see also [24]) and the other part $\partial V_{u}$ is subjected to the prescribed displacements $\mathbf{u}^{d}$.

From $\mathbf{T}=\mathbf{T}^{\text {sym }}+\mathbf{T}^{\text {skew }}$ with $T_{i j}{ }^{\text {skew }}=\frac{1}{2} e_{i j k} e_{m n k} T_{m n}$ and using eqs. 46 and 47 , a single equilibrium equation involving $\mathbf{T}^{\text {skew }}$ and $\mathbf{m}^{D}$ can be written as follows:

$$
\operatorname{div} \mathbf{T}^{\text {sym }}+\frac{1}{2} \operatorname{curl}\left(\operatorname{div} \mathbf{m}^{D}\right)=0 \text { in } V
$$

Using the Stokes-Helmoltz decomposition (equations 19 and 28), the equilibrium equation (48) together with the constitutive relationships can be rewritten in the form of a partial differential equation of Naviertype in $V$ :

$$
\operatorname{div} \mathbf{C}: \boldsymbol{\varepsilon}_{e}^{\|}+\frac{1}{2} \operatorname{curl}\left(\operatorname{div}\left(\mathbf{A}: \boldsymbol{\kappa}_{e}^{D \|}\right)\right)+\mathbf{f}^{\perp}=0,
$$

where $\boldsymbol{\varepsilon}_{e}^{\|}, \boldsymbol{\kappa}_{e}^{D \|}$ are respectively given by:

$$
\begin{array}{r}
\varepsilon_{i j}^{e, \|}=\frac{1}{2}\left(w_{i, j}+w_{j, i}\right) \\
\kappa_{i j}^{e D, \|}=\frac{1}{2} e_{i k l} w_{l, k j}
\end{array}
$$

and where the incompatible fictive body force density arising from the generalized defects is given by:

$$
\mathbf{f}^{\perp}=\operatorname{div} \mathbf{C}: \boldsymbol{\varepsilon}_{e}^{\perp}+\frac{1}{2} \operatorname{curl}\left(\operatorname{div}\left(\mathbf{A}: \boldsymbol{\kappa}_{e}^{D, \perp}\right)\right)
$$


with $\varepsilon_{i j}^{e, \perp}=\frac{1}{2}\left(U_{i j}^{e, \perp}+U_{j i}^{e, \perp}\right)$ and with $\kappa_{i j}^{e, \perp}=-\frac{1}{2} e_{m n i} G_{m n j}^{e, s k e w, \perp}$. Eqs. (49) to (52) together with adequate boundary conditions on displacements, force and couple traction vectors $[34,24]$ set a couple stress elasticity problem for the unknown fields $\mathbf{w}, \boldsymbol{\varepsilon}_{e}^{\|}$and $\boldsymbol{\kappa}_{e}^{D, \|}$ which can therefore be determined uniquely. The incompatible volume fictive body force $\mathbf{f}^{\perp}$ is first determined by solving the Poisson-type equations $(24,31)$ for $\mathbf{G}_{e}^{\perp}, \mathbf{U}_{e}^{\perp}$ to give $\boldsymbol{\kappa}_{e}^{D, \perp}$ and $\boldsymbol{\varepsilon}_{e}^{\perp}$ after defect density fields $(\boldsymbol{\alpha}, \boldsymbol{\pi})$ have been initially prescribed.

Assuming a homogeneous reference medium with uniform elastic moduli and couple stress moduli $C_{i j k l}^{0}$ and $A_{i j k l}^{0}$, such that $C_{i j k l}=C_{i j k l}^{0}$ and $A_{i j k l}=A_{i j k l}^{0}$, no "polarization tensor" fields are accounted for (i.e. no iterative scheme is needed). Therefore, eqs.49 to 52 yield, in component form:

$$
C_{i j k l}^{0} w_{k, l j}+\frac{1}{4} e_{i k l} e_{p r s} A_{l m p q}^{0} w_{s, r q m k}+C_{i j k l}^{0} \varepsilon_{k l, j}^{e, \perp}+\frac{1}{2} e_{i k l} A_{l m p q}^{0} \kappa_{p q, m k}^{e D, \perp}=0
$$

Using eq. 42 and eq. 43 in eq.53 yields:

$$
\begin{aligned}
& \mu w_{i, k k}+(\lambda+\mu) w_{k, k i}+\mu l^{2}\left(w_{k, i k m m}-w_{i, k k m m}\right) \\
& +\lambda \varepsilon_{k k, i}^{e, \perp}+2 \mu \varepsilon_{i k, k}^{e, \perp}+2 \mu l^{2} e_{i k l} \kappa_{l m, m k}^{e D, \perp}-\frac{A_{2}}{2} e_{i k l} \kappa_{m l, m k}^{e D, \perp}=0
\end{aligned}
$$

It is noteworthy that the classic size-independent theory with incompatibilities described in [6] for instance is found with $l=0$ and $A_{2}=0$. In this case, the term containing the incompatible elastic curvature fields due to g-disclinations as well as that with compatible elastic curvatures vanish and the classic Navier-type operator including only second order partial spatial derivatives with classic isotropic linear elasticity is retrieved.

\section{Fourier method}

\subsection{Solution of Poisson-type equations in Fourier space}

The previous Poisson and Navier-type equations can be solved using the Fourier Transform method. Indeed, the unknown vector field $\mathbf{w}(\boldsymbol{x})$ can be obtained by using the spectral method based on Fourier transforms to derive later on the stresses, elastic rotations etc. in the Fourier space. Then, the elastic fields are estimated in the real space using the inverse Fourier Transform. The FFT algorithm is well suited for periodic media. This one will be developed in section 6 to estimate the discrete Fourier transforms on FFT grids.

In the Fourier space, let $\boldsymbol{\xi}$ be the Fourier vector of magnitude $\xi=\sqrt{\boldsymbol{\xi} \cdot \boldsymbol{\xi}}$ and components $\xi_{i}$ in a cartesian coordinate system in a general three-dimensional setting. The complex imaginary number is denoted $\mathrm{i}$ and defined as $\mathrm{i}=\sqrt{-1}$.

Let $\widetilde{\boldsymbol{\alpha}}(\boldsymbol{\xi}), \widetilde{\mathbf{U}}_{e}^{\perp}(\boldsymbol{\xi}), \widetilde{\boldsymbol{\varepsilon}}_{e}^{\perp}(\boldsymbol{\xi}), \widetilde{\boldsymbol{\pi}}(\boldsymbol{\xi}), \widetilde{\mathbf{G}}_{e}^{\perp}(\boldsymbol{\xi})$ and $\widetilde{\boldsymbol{\kappa}}_{e}^{D, \perp}(\boldsymbol{\xi})$ be the continuous Fourier transforms of $\boldsymbol{\alpha}(\boldsymbol{x})$, $\mathbf{U}_{e}^{\perp}(\boldsymbol{x}), \boldsymbol{\varepsilon}_{e}^{\perp}(\boldsymbol{x}), \boldsymbol{\pi}(\boldsymbol{x}), \mathbf{G}_{e}^{\perp}(\boldsymbol{x})$ and $\boldsymbol{\kappa}_{e}^{D, \perp}(\boldsymbol{x})$. Then, the Poisson-type equations (eqs. 24 and 31) are solved using the differentiation theorem in Fourier space. Using component notations, Eq. 26 writes in the Fourier space

$$
\begin{aligned}
& \widetilde{G}_{i j k}^{e, \perp}(\boldsymbol{\xi})=\frac{\mathrm{i}}{\xi^{2}} \xi_{l} e_{k l m} \widetilde{\pi}_{i j m}(\boldsymbol{\xi}) \forall \boldsymbol{\xi} \neq \mathbf{0} \\
& \widetilde{G}_{i j k}^{e, \perp}(\mathbf{0})=\mathbf{0}
\end{aligned}
$$

and Eq. 33 yields in the Fourier space

$$
\begin{aligned}
& \widetilde{U}_{i j}^{e, \perp}(\boldsymbol{\xi})=\frac{\mathrm{i}}{\xi^{2}} \xi_{k}\left(e_{j k l} \widetilde{\alpha}_{i l}(\boldsymbol{\xi})+\widetilde{G}_{i j k}^{e, \perp}(\boldsymbol{\xi})-\widetilde{G}_{i k j}^{e, \perp}(\boldsymbol{\xi})\right) \forall \boldsymbol{\xi} \neq \mathbf{0} \\
& \widetilde{U}_{i j}^{e, \perp}(\mathbf{0})=\mathbf{0}
\end{aligned}
$$

Therefore, $\widetilde{\boldsymbol{\varepsilon}}_{e}^{\perp}(\boldsymbol{\xi})$ is derived from the symmetric part of $\widetilde{\mathbf{U}}_{e}^{\perp}(\boldsymbol{\xi})$ and $\widetilde{\boldsymbol{\kappa}}_{e}^{D, \perp}(\boldsymbol{\xi})$ is obtained from the skewsymmetric part of $\widetilde{\mathbf{G}}_{e}^{\perp}(\boldsymbol{\xi})$ (see eq. 40 ). 


\subsection{Solution of Navier-type equation in Fourier space}

Let $\widetilde{\mathbf{w}}(\boldsymbol{\xi}), \widetilde{\boldsymbol{\varepsilon}}_{e}^{\|}(\boldsymbol{\xi})$ and $\widetilde{\boldsymbol{\kappa}}_{e}^{D, \|}(\boldsymbol{\xi})$ be the continous Fourier transform of $\mathbf{w}(\boldsymbol{x}), \boldsymbol{\varepsilon}_{e}^{\|}(\boldsymbol{x})$ and $\boldsymbol{\kappa}_{e}^{D, \|}(\boldsymbol{x})$. Then, the Fourier transform of the Navier-type equation (equation 53) yields

$$
C_{i j k l}^{0} \xi_{l} \xi_{j} \widetilde{w}_{k}(\boldsymbol{\xi})-\frac{1}{4} e_{i k l} e_{p r s} A_{l m p q}^{0} \xi_{r} \xi_{q} \xi_{m} \xi_{k} \widetilde{w}_{s}(\boldsymbol{\xi})=\mathrm{i} C_{i j k l}^{0} \xi_{j} \widetilde{\varepsilon}_{k l}^{e, \perp}(\boldsymbol{\xi})+\frac{1}{2} e_{i k l} A_{l m p q}^{0} \xi_{m} \xi_{k} \widetilde{\kappa}_{p q}^{e D, \perp}(\boldsymbol{\xi})
$$

The solution $\widetilde{\mathbf{w}}(\boldsymbol{\xi})$ can be obtained with the introduction of the Green tensor $\widetilde{G}_{i k}(\boldsymbol{\xi})$ in the Fourier space:

$$
\widetilde{w}_{i}(\boldsymbol{\xi})=\widetilde{G}_{i k}(\boldsymbol{\xi}) \widetilde{f}_{k}^{\perp}(\boldsymbol{\xi})
$$

where:

$$
\widetilde{G}_{i k}(\boldsymbol{\xi})=\left(C_{i j k l}^{0} \xi_{l} \xi_{j}-\frac{1}{4} e_{i j l} A_{l m p q}^{0} e_{p r k} \xi_{r} \xi_{q} \xi_{m} \xi_{j}\right)^{-1}
$$

and:

$$
\widetilde{f}_{i}^{\perp}(\boldsymbol{\xi})=\mathrm{i} C_{i j k l}^{0} \xi_{j} \widetilde{\varepsilon}_{k l}^{e, \perp}(\boldsymbol{\xi})+\frac{1}{2} e_{i k l} A_{l m p q}^{0} \xi_{m} \xi_{k} \widetilde{\kappa}_{p q}^{e D, \perp}(\boldsymbol{\xi})
$$

It is noteworthy that eqs. 58 to 60 can be applied to any centrosymmetric anisotropic couple stress materials.

For isotropic centrosymmetric couple stress materials, $C_{i j k l}^{0}$ and $A_{i j k l}^{0}$ are defined by eqs. 38 and 39 . In this case, the expression of the non local Green tensor can be found in $[48,56]$. Thus, the expression of $\widetilde{G}_{i k}(\boldsymbol{\xi})$ is given by:

$$
\begin{aligned}
& \widetilde{G}_{i k}(\boldsymbol{\xi})=\frac{1}{\mu \xi^{2}}\left[\frac{1}{1+l^{2} \xi^{2}}\left(\delta_{i k}-\frac{\xi_{i} \xi_{k}}{\xi^{2}}\right)+\frac{\mu}{\lambda+2 \mu} \frac{\xi_{i} \xi_{k}}{\xi^{2}}\right] \forall \boldsymbol{\xi} \neq \mathbf{0} \\
& \widetilde{G}_{i k}(\mathbf{0})=\mathbf{0}
\end{aligned}
$$

The compatible elastic strain $\widetilde{\boldsymbol{\varepsilon}}_{e}^{\|}(\boldsymbol{\xi})$ is obtained in the Fourier space from the differentiation rule:

$$
\begin{aligned}
& \widetilde{\varepsilon}_{i j}^{e, \|}(\boldsymbol{\xi})=\frac{1}{2} \mathrm{i}\left(\xi_{j} \widetilde{w}_{i}(\boldsymbol{\xi})+\xi_{i} \widetilde{w}_{j}(\boldsymbol{\xi})\right) \\
& \widetilde{\boldsymbol{\kappa}}_{i j}^{e D, \|}(\boldsymbol{\xi})=-\frac{1}{2} e_{i k l} \xi_{j} \xi_{k} \widetilde{w}_{l}(\boldsymbol{\xi})
\end{aligned}
$$

\subsection{Stress and couple stress fields}

Knowing $\widetilde{\mathbf{U}}_{e}^{\perp}(\boldsymbol{\xi})$ and $\widetilde{\mathbf{U}}_{e}^{\|}(\boldsymbol{\xi})$, the (total) elastic distortion in the Fourier space yields

$$
\widetilde{U}_{i j}^{e}=\widetilde{U}_{i j}^{e, \perp}+\widetilde{U}_{i j}^{e, \|}
$$

The stress $\widetilde{\mathbf{T}}(\boldsymbol{\xi})$ in Fourier space is obtained in component form as:

$$
\begin{aligned}
& \widetilde{T}_{i j}(\boldsymbol{\xi})=C_{i j k l}^{0} \widetilde{\varepsilon}_{k l}^{e}(\boldsymbol{\xi}) \forall \boldsymbol{\xi} \neq \mathbf{0} \\
& \widetilde{T}_{i j}(\mathbf{0})=0
\end{aligned}
$$

where $\widetilde{\varepsilon}_{i j}^{e}=\frac{1}{2}\left(\widetilde{U}_{i j}^{e}+\widetilde{U}_{j i}^{e}\right)$ and where the far-field (overall) stress which is the spatial average of $T_{i j}$ over the periodic unit cell is set to zero. Here, only the internal stress field will be computed in section 7 .

The couple stress $\widetilde{\mathbf{m}^{\mathbf{D}}}(\boldsymbol{\xi})$ in Fourier space is obtained in component form as:

$$
\begin{aligned}
& \widetilde{m}_{i j}^{D}(\boldsymbol{\xi})=A_{i j k l}^{0} \widetilde{\kappa}_{k l}^{e D}(\boldsymbol{\xi}) \forall \boldsymbol{\xi} \neq \mathbf{0} \\
& \widetilde{m}_{i j}^{D}(\mathbf{0})=0
\end{aligned}
$$


where the far-field (overall) deviatoric couple stress which is taken as the spatial average of $m_{i j}^{D}$ over the periodic unit cell is set to zero. Here only the internal couple stress field due to generalized defects will be computed in section 7 .

The elastic stress and couple stress moduli $C_{i j k l}^{0}$ and $A_{i j k l}^{0}$ in eq. 64 and 65 are defined by eqs. 38 and 39. Then, the inverse Fourier transforms of $\widetilde{\mathbf{T}}(\boldsymbol{\xi})$ and $\widetilde{\mathbf{m}^{D}}(\boldsymbol{\xi})$ are numerically computed using the FFT algorithm and inverse FFT allows finding $\mathbf{T}$ and $\mathbf{m}^{D}$ on the discretized periodic unit cell.

\section{Fast Fourier Transform numerical implementation}

\subsection{Discrete Fourier Transforms and FFT}

The field equations derived in the Fourier space are now solved by 2D discrete Fourier transforms with the Fast Fourier Transform (FFT) algorithm. Here, periodicity is assumed for the distribution of g-disclination densities (i.e. $\pi$ ), with spatial periods $T_{1}$ and $T_{2}$ in the $x_{1}$ and $x_{2}$ directions, respectively. The periodic representative volume element (RVE) or unit cell is discretized by a regular rectangular grid with $N_{1} \times N_{2}$ pixels with position vector $\boldsymbol{x}=\left((i-1) \delta_{1},(j-1) \delta_{2}\right)$, where $i=1 \rightarrow N_{1}, j=1 \rightarrow N_{2}$ and $\delta_{1}, \delta_{2}$ are the pixel sizes in the $x_{1}$ and $x_{2}$ directions with $\delta_{1}=\delta_{2}=\delta$. The total number of FFT grid points is $N_{\text {tot }}=N_{1} \times N_{2}$. Here, the FFTW package of Matlab is used to compute discrete Fourier transforms [17]. The discrete FFT of a given spatial function $f$ is $\widehat{f}=\operatorname{FFT}(f)$. The inverse Fourier transform is $f=\mathrm{FFT}^{-1}(\widehat{f})$. They write with the Matlab FFT convention:

$$
\widehat{f}(k, l)=\sum_{i=1}^{N 1} \sum_{j=1}^{N 2} f(i, j) \exp \left(-2 \pi \mathrm{i}\left(\frac{(i-1)(k-1)}{N 1}+\frac{(j-1)(l-1)}{N 2}\right)\right)
$$

and

$$
f(i, j)=\frac{1}{N_{\text {tot }}} \sum_{k=1}^{N 1} \sum_{l=1}^{N 2} \widehat{f}(k, l) \exp \left(+2 \pi \mathrm{i}\left(\frac{(i-1)(k-1)}{N 1}+\frac{(j-1)(l-1)}{N 2}\right)\right)
$$

It should be pointed out that Eqs. 66 and 67 are finite sums which can be determined exactly by FFT for periodic unit cells.

\subsection{DFT differentiation rules based on centered finite difference approximation}

Here, the following differentiation rules are used for first-, second- and fourth- order partial derivatives calculated on the discrete grid based on a 9-pixel centered finite difference approximation [45]: 


$$
\begin{aligned}
& \frac{\partial f(i, j)}{\partial x_{1}}=\frac{f(i+1, j)-f(i-1, j)}{2 \delta_{1}} \\
& \frac{\partial f(i, j)}{\partial x_{2}}=\frac{f(i, j+1)-f(i, j-1)}{2 \delta_{2}} \\
& \frac{\partial^{2} f(i, j)}{\partial x_{1}^{2}}=\frac{f(i+1, j)-2 f(i, j)+f(i-1, j)}{\delta_{1}^{2}} \\
& \frac{\partial^{2} f(i, j)}{\partial x_{2}^{2}}=\frac{f(i, j+1)-2 f(i, j)+f(i, j-1)}{\delta_{2}^{2}} \\
& \frac{\partial^{2} f(i, j)}{\partial x_{1} \partial x_{2}}=\frac{f(i+1, j+1)+f(i-1, j-1)}{4 \delta_{1} \delta_{2}} \\
& -\frac{f(i+1, j-1)+f(i-1, j+1)}{4 \delta_{1} \delta_{2}} \\
& \frac{\partial^{4} f(i, j)}{\partial x_{1}^{4}}=\frac{f(i-2, j)-4 f(i-1, j)+6 f(i, j)-4 f(i+1, j)+f(i+2, j)}{\delta_{1}^{4}} \\
& \frac{\partial^{4} f(i, j)}{\partial x_{2}^{4}}=\frac{f(i, j-2)-4 f(i, j-1)+6 f(i, j)-4 f(i, j+1)+f(i, j+2)}{\delta_{2}^{4}} \\
& \frac{\partial^{4} f(i, j)}{\partial x_{1}^{2} \partial x_{2}^{2}}=\frac{4 f(i, j)}{\delta_{1}^{2} \delta_{2}^{2}} \\
& -2 \frac{f(i-1, j)+f(i+1, j)+f(i, j-1)+f(i, j+1)}{\delta_{1}^{2} \delta_{2}^{2}} \\
& +\frac{f(i+1, j-1)+f(i-1, j-1)}{\delta_{1}^{2} \delta_{2}^{2}} \\
& +\frac{f(i+1, j+1)+f(i-1, j+1)}{\delta_{1}^{2} \delta_{2}^{2}} \\
& \frac{\partial^{4} f(i, j)}{\partial x_{2} \partial x_{1}^{3}}=\frac{f(i+2, j+1)+2 f(i-1, j+1)}{4 \delta_{1}^{3} \delta_{2}} \\
& -\frac{f(i-2, j+1)+2 f(i+1, j+1)}{4 \delta_{1}^{3} \delta_{2}} \\
& +\frac{f(i-2, j-1)+2 f(i+1, j-1)}{4 \delta_{1}^{3} \delta_{2}} \\
& -\frac{f(i+2, j-1)+2 f(i-1, j-1)}{4 \delta_{1}^{3} \delta_{2}} \\
& \frac{\partial^{4} f(i, j)}{\partial x_{1} \partial x_{2}^{3}}=\frac{f(i+1, j+2)+2 f(i+1, j-1)}{4 \delta_{1} \delta_{2}^{3}} \\
& -\frac{f(i-1, j+2)+2 f(i+1, j+1)}{4 \delta_{1} \delta_{2}^{3}} \\
& +\frac{f(i-1, j-2)+2 f(i-1, j+1)}{4 \delta_{1} \delta_{2}^{3}} \\
& -\frac{f(i-1, j+2)+2 f(i-1, j-1)}{4 \delta_{1} \delta_{2}^{3}}
\end{aligned}
$$

Using Eqs. 66 to 88, the subtitutions due to correspondance between continuous and discrete Fourier transform derivatives are the following:

$$
\mathrm{i} \xi_{1} \leftrightarrow \frac{\mathrm{i}}{\delta_{1}} \sin \left(\frac{2 \pi(k-1)}{N 1}\right)
$$




$$
\begin{gathered}
\mathrm{i} \xi_{2} \leftrightarrow \frac{\mathrm{i}}{\delta_{2}} \sin \left(\frac{2 \pi(l-1)}{N 2}\right) \\
-\xi_{1}^{2} \leftrightarrow \frac{2}{\delta_{1}^{2}}\left(\cos \left(\frac{2 \pi(k-1)}{N 1}\right)-1\right) \\
-\xi_{2}^{2} \leftrightarrow \frac{2}{\delta_{2}^{2}}\left(\cos \left(\frac{2 \pi(l-1)}{N 2}\right)-1\right) \\
-\xi_{1} \xi_{2} \leftrightarrow \frac{1}{2 \delta_{1} \delta_{2}} \cos \left(2 \pi\left(\frac{(k-1)}{N 1}+\frac{(l-1)}{N 2}\right)\right) \\
-\frac{1}{2 \delta_{1} \delta_{2}} \cos \left(2 \pi\left(\frac{(k-1)}{N 1}-\frac{(l-1)}{N 2}\right)\right) \\
\xi_{1}^{4} \leftrightarrow \frac{4}{\delta_{1}^{4}}\left(\cos \left(\frac{2 \pi(k-1)}{N 1}\right)-1\right)^{2} \\
\xi_{2}^{4} \leftrightarrow \frac{4}{\delta_{2}^{4}}\left(\cos \left(\frac{2 \pi(l-1)}{N 2}\right)-1\right)^{2} \\
\xi_{1}^{2} \xi_{2}^{2} \leftrightarrow \frac{4}{\delta_{1}^{2} \delta_{2}^{2}}\left(\cos \left(\frac{2 \pi(k-1)}{N 1}\right)-1\right)\left(\cos \left(\frac{2 \pi(l-1)}{N 2}\right)-1\right) \\
\xi_{1}^{3} \xi_{2} \leftrightarrow \frac{2}{\delta_{1}^{3} \delta_{2}} \sin \left(2 \pi\left(\frac{(k-1)}{N 1}\right)\right) \sin \left(2 \pi\left(\frac{(l-1)}{N 2}\right)\right) \\
\times\left(1-\cos \left(\frac{2 \pi(k-1)}{N 1}\right)\right) \\
\xi_{1} \xi_{2}^{3} \leftrightarrow \frac{2}{\delta_{1} \delta_{2}^{3}} \sin \left(2 \pi\left(\frac{(k-1)}{N 1}\right)\right) \sin \left(2 \pi\left(\frac{(l-1)}{N 2}\right)\right) \\
\times\left(1-\cos \left(\frac{2 \pi(l-1)}{N 2}\right)\right)
\end{gathered}
$$

In section 7 of the present paper, the 9-pixel approximation is sufficient to give accurate enough results for strong gradients of stress/couple stress fields of g-disclinations in comparison with existing analytical solutions . Higher order pixel approximations may also be developed [41] to further refine the FFT analysis. In [6], it was shown that the present FFT method with centered-difference based-DFT avoids spurious Gibbs oscillations occuring with classic FFT techniques, especially when defect densities are prescribed to a single pixel.

\section{Application to infinite straight wedge disclinations}

\subsection{Materials and numerical data}

In the forthcoming applications, the g-disclination densities are prescribed using a regular Gaussian function. 2D FFT $N \times N$ square grids with $\delta_{1}=\delta_{2}=\delta, N=N_{1}=N_{2}$ and $N_{t o t}=N^{2}$ are considered. In this section, the FFT grid will be set to $1024 \times 1024$ pixels with pixel size: $\delta=0.1 b$ where $b$ is the magnitude of the Burgers vector. Here, a face-centered cubic metal like Copper is studied, for which the lattice parameter is $a_{0}=0.36151 \mathrm{~nm}$. The isotropic elastic constants of Copper $(\mathrm{Cu})$ will be used for the simulations: $\mu=47800 \mathrm{MPa}, v=0.34$. The magnitude of the Burgers vector of $\mathrm{Cu}$ is $b=\sqrt{2} a_{0} / 2$, i.e. $b=0.25563 \mathrm{~nm}$. Following [32] for dislocations, [51], and [49,16] for disclinations the length scale $l$ is set to $b / 2$ to make $A_{1} \approx \mu b^{2}$. For wedge disclinations, it will be seen that the term containing $A 2$ in eq. 54 vanishes.

In [6], the stresses for both pure screw and edge dislocations were already computed to assess the present numerical spectral method by comparing the FFT solutions to analytical expressions $[20,1]$ and finite ele- 
ment results. Here, numerical FFT results for disclinations, disclination dipole and walls will be compared to analytical results reported in [5], [10], [46].

\subsection{Two-dimensional equations for g-disclinations}

In the following applications, we consider straight g-disclinations such that the defect line lies along the $\mathbf{e}_{3}$ axis. Thus, the elastic fields are invariant with respect to $x_{3}$. Here, the defect is based on elastic distortion discontinuities described by non zero $\llbracket U_{12}^{e} \rrbracket$ and $\llbracket U_{21}^{e} \rrbracket$. Thus, in this case, eqs. 16 and 17 simplify into

$$
\begin{aligned}
& \int_{S} \pi_{123} d S=\int_{S}\left(G_{122,1}^{e, \perp}-G_{121,2}^{e, \perp}\right) d S=\llbracket U_{12}^{e} \rrbracket \\
& \int_{S} \pi_{213} d S=\int_{S}\left(G_{212,1}^{e, \perp}-G_{211,2}^{e, \perp}\right) d S=\llbracket U_{21}^{e} \rrbracket
\end{aligned}
$$

Consequently, given $\pi_{123}(\boldsymbol{x})$ and $\pi_{213}(\boldsymbol{x})$, the incompatible elastic 2-distortions are solutions of the four following Poisson-type equations (see eq.26)

$$
\begin{aligned}
& G_{122,11}^{e, \perp}+G_{122,22}^{e, \perp}=\pi_{123,1} \\
& G_{121,11}^{e, \perp}+G_{121,22}^{e, \perp}=-\pi_{123,2} \\
& G_{212,11}^{e, \perp}+G_{212,22}^{e, \perp}=\pi_{213,1} \\
& G_{211,11}^{e, \perp}+G_{211,22}^{e, \perp}=-\pi_{213,2}
\end{aligned}
$$

Once $G_{122}^{e, \perp}, G_{121}^{e, \perp}, G_{212}^{e, \perp}$ and $G_{211}^{e, \perp}$ are obtained, four other Poisson-type equations are needed to find in turn the incompatible elastic 1-distortions $U_{11}^{e, \perp}, U_{22}^{e, \perp}, U_{12}^{e, \perp}$ and $U_{21}^{e, \perp}$ using eq. 33 (without dislocation densities)

$$
\begin{aligned}
& U_{12,11}^{e, \perp}+U_{12,22}^{e, \perp}=-G_{121,1}^{e, \perp} \\
& U_{11,11}^{e, \perp}+U_{11,22}^{e, \perp}=G_{121,2}^{e, \perp} \\
& U_{21,11}^{e, \perp}+U_{21,22}^{e, \perp}=-G_{212,2}^{e, \perp} \\
& U_{22,11}^{e, \perp}+U_{22,22}^{e, \perp}=G_{212,1}^{e, \perp}
\end{aligned}
$$

All the previous equations will be solved successively in the Fourier space for the particular cases of straight wedge disclination and wedge disclination dipole. Then, the generalized Navier-type equation is solved in the Fourier space for the compatible 1- and 2-elastic distortions (compatible elastic strain and elastic curvature tensors).

\subsection{Single straight wedge disclination}

First, the case of a pure straight wedge disclination is considered. This corresponds to an elastic distortion discontinuity in the negative half-plane $\left(x_{1}=0, x_{2} \leq 0\right)$. The only non zero discontinuities are $\llbracket U_{12}^{e} \rrbracket=$ $\llbracket \omega_{12}^{e} \rrbracket=-\llbracket \Omega_{3}^{e} \rrbracket$ and $\llbracket U_{21}^{e} \rrbracket=\llbracket \omega_{21}^{e} \rrbracket=+\llbracket \Omega_{3}^{e} \rrbracket$, where $\llbracket \Omega_{3}^{e} \rrbracket=\Omega_{3+}^{e}-\Omega_{3-}^{e}$ is the elastic rotation discontinuity along the $\mathbf{e}_{3}$ axis (the domains (+) and (-) respectively correspond to $x_{1}>0$ and $x_{1}<0$ ). This g-disclination is equivalent to a pure disclination with the positive Frank vector component along the $\mathbf{e}_{3}$ axis [46] with $\llbracket \Omega_{3}^{e} \rrbracket=\omega$. Thus, the discontinuity in the elastic distortion is such that

$$
\llbracket \Omega_{3}^{e} \rrbracket=\omega=\int_{S} \pi_{213} d S
$$

where $\pi_{213}=-\pi_{123}$. Thus, only two Poisson equations containing $\pi_{213}$ are considered and solved in the Fourier space. For the simulations, the g-disclination density follows a Gaussian distribution: 


$$
\begin{aligned}
& \pi_{213}(\boldsymbol{x})=\frac{\omega}{2 \pi \sigma^{2}} \exp \left(-\frac{r^{2}}{2 \sigma^{2}}\right) \text { if } r \leq r_{0} \\
& \pi_{213}(\boldsymbol{x})=0 \text { if } r>r_{0}
\end{aligned}
$$

with $r=\sqrt{x_{1}^{2}+x_{2}^{2}}$ and $\sigma=0.1 r_{0}$.

Then, the non zero incompatible elastic curvature components (equivalent to incompatible elastic 2distortions) are obtained as

$$
\begin{aligned}
& \kappa_{31}^{e, \perp}=-G_{121}^{e, \perp} \\
& \kappa_{32}^{e, \perp}=-G_{122}^{e, \perp}
\end{aligned}
$$

By solving the non zero incompatible and compatible elastic curvatures, the elastic curvatures $\kappa_{32}^{e}=$ $\kappa_{32}^{e, \perp}+\kappa_{32}^{e, \|}$ and $\kappa_{31}^{e}=\kappa_{31}^{e, \perp}+\kappa_{31}^{e, \|}$ are computed by inverse FFT on a $2 \mathrm{D}$ grid with $r_{0}=0.5 b$ and $\omega=5 / 6$ rad. Therefore, the $A 2$ term in eq. 54 vanishes because of invariance of incompatible elastic curvature along $x_{3}$ together with only non zero $\kappa_{32}^{e, \perp}$ and $\kappa_{31}^{e, \perp}$ for this particular $2 \mathrm{D}$ case. The numerical results are reported in Fig. 2. The results show that the respective variations of $\kappa_{32}^{e}$ and $\kappa_{31}^{e}$ along $x_{1}$ and $x_{2}$ match exactly the analytical solutions of [10] or [5]:

$$
\begin{aligned}
\kappa_{31}^{e} & =-\frac{\llbracket \Omega_{3}^{e} \rrbracket}{2 \pi} \frac{x_{2}}{r^{2}} \\
\kappa_{32}^{e} & =\frac{\llbracket \Omega_{3}^{e} \rrbracket}{2 \pi} \frac{x_{1}}{r^{2}}
\end{aligned}
$$
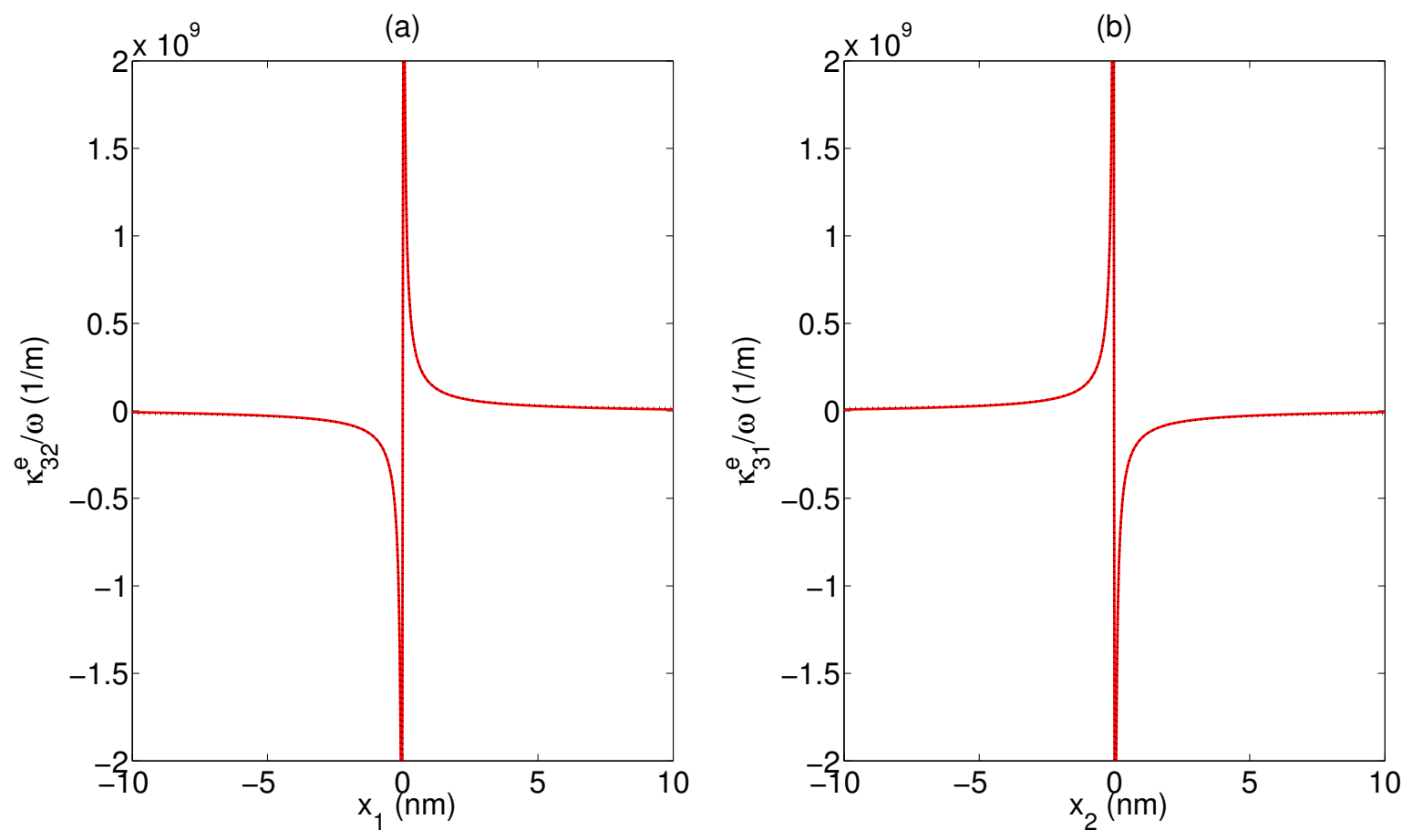

Fig. 2 Elastic curvatures for a single straight wedge disclination: (a) $\kappa_{32}^{e}$, (b) $\kappa_{31}^{e}$ normalized by $\omega=\llbracket \Omega_{3}^{e} \rrbracket$. Comparisons with the solutions given by $[5,10]$ (dashed lines).

Once the elastic curvatures are calculated, the non zero couple stress components $m_{31}^{D}$ and $m_{32}^{D}$ are obtained using the constitutive relationship (eq. 43), see Fig. 3. The results are consistent with the analytical 
solutions obtained with a couple stress theory with disclinations [5]. Couple stress contours for $m_{31}^{D}$ and $m_{32}^{D}$ are also reported in Fig. 4.
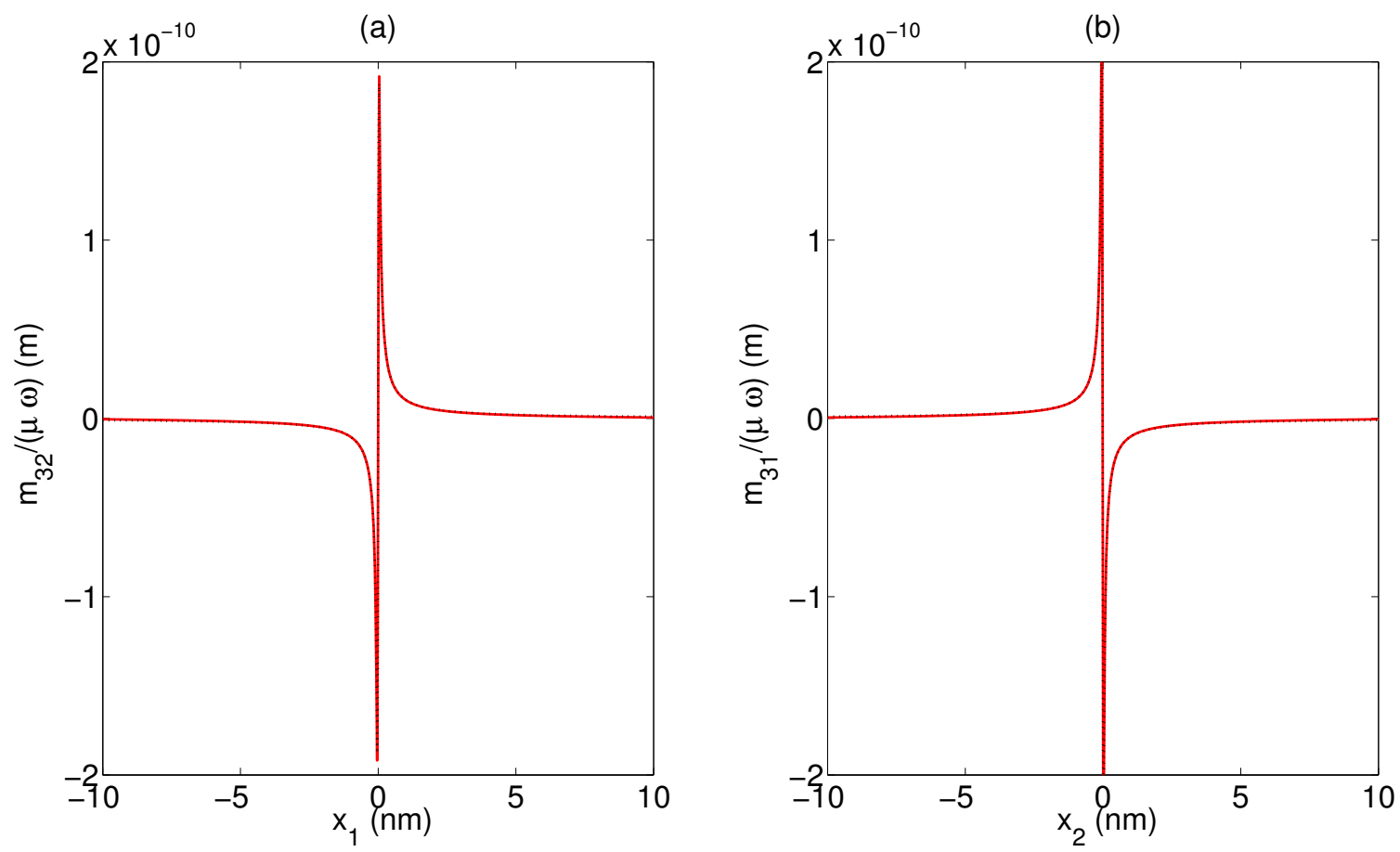

Fig. 3 Couple stresses for a single straight wedge disclination: $m_{32}^{D}$ (a), $m_{31}^{D}$ (b) non zero couple stress components normalized by $\mu \omega$. Comparisons with the solutions given by [5] (dashed lines). 


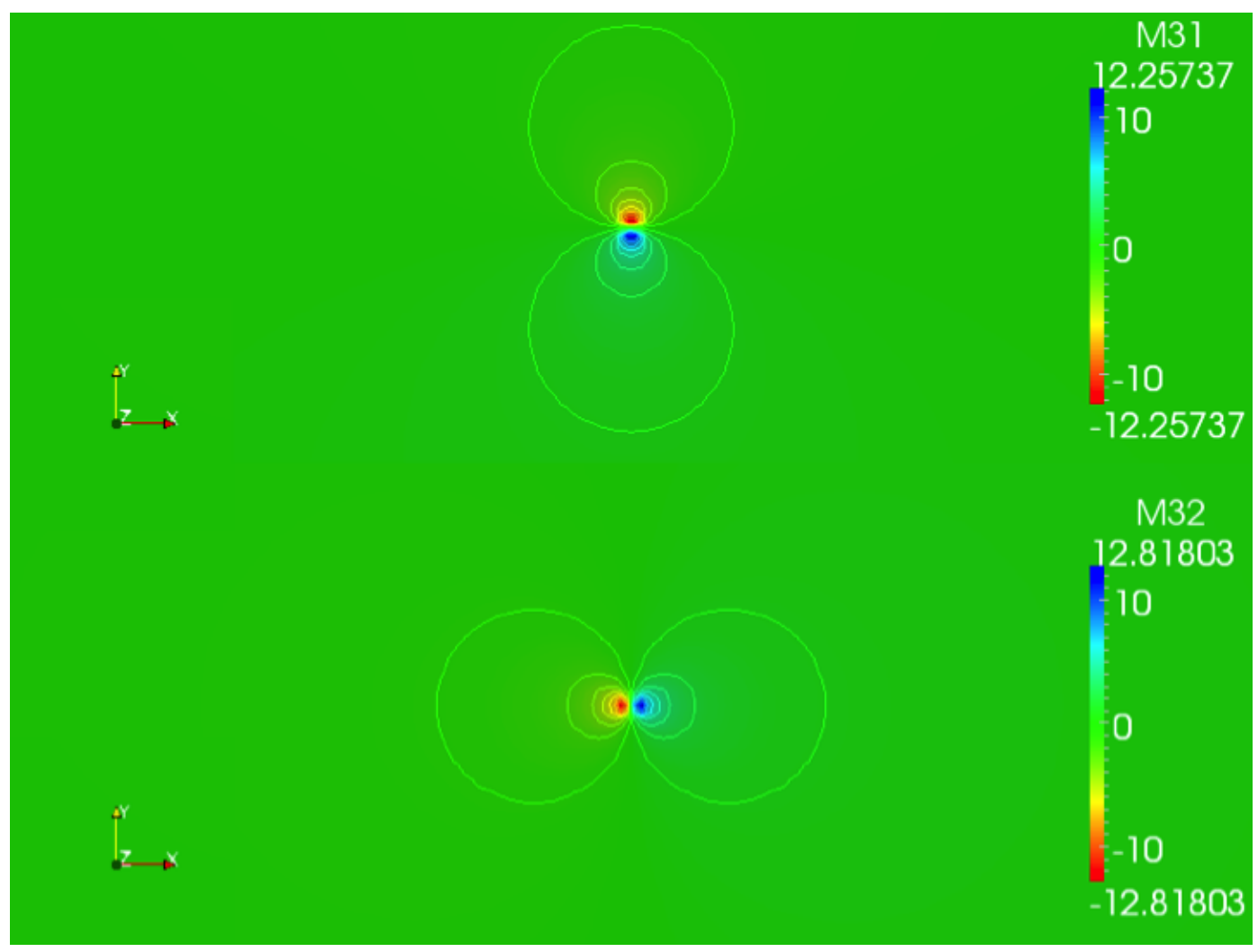

Fig. 4 FFT simulations of couple stresses for a single straight wedge disclination: $m_{31}^{D}$ (top), $m_{32}^{D}$ (bottom) non zero couple stress components normalized by $\omega=\llbracket \Omega_{3}^{e} \rrbracket$.

\subsection{Straight wedge disclination dipole}

A second application is the case of a wedge disclination dipole as described in Fig. 5 which is commonly used to describe tilt grain boundaries as originally proposed by [31] and later improved by Gertsman et al. [18, 39]. In this representation, the grain boundary is represented in the form of a complex arrangement of periodic disclination dipole walls associated with the minority structural units (see Fig. 6).

For a pure wedge disclination dipole, the positive (resp. negative) pole is distributed by using the same Gaussian distribution as in eq. 110 at location $\left(x_{1}=0, x_{2}=+a\right)\left(\right.$ resp. $\left.\left(x_{1}=0, x_{2}=-a\right)\right)$ with disclination strength $\llbracket \Omega_{3}^{e} \rrbracket$ (resp. $\left.-\llbracket \Omega_{3}^{e} \rrbracket\right)$.

In order to get stress fields similar to that of an equivalent straight edge dislocation, the semi-length of the dipole is set to $a=b /\left(2 \llbracket \Omega_{3}^{e} \rrbracket\right)$ (see e.g. [46]) with $\llbracket \Omega_{3}^{e} \rrbracket=5 / 6 \mathrm{rad}$. For the FFT simulations, the stress components are obtained by inverse FFT on the 2D grid after successively computing in the discrete Fourier space the incompatible elastic curvatures, the incompatible elastic 1-distortions, the compatible elastic distortions and the stresses and couple stresses using the constitutive relationships.

Fig. 7 displays the stress components $T_{11}, T_{12}$ obtained by FFT and normalized by $D \omega$ where $\omega=$ $\llbracket \Omega_{3}^{e} \rrbracket$ and $D=\mu /(2 \pi(1-v))$. Fig. 8 describes the couple stress components $m_{31}^{D}$ and $m_{32}^{D}$ obtained by FFT and normalized by $\mu \omega$. Excellent agreement is found with the analytical stress components given by $[10,46,5]$ for wedge disclination dipoles. Normal and shear stresses contour plots normalized by $D \omega$ $(D=\mu /(2 \pi(1-v)))$ are reported in Fig. 9 and couple stress plots normalized by $\omega$ are reported in Fig. 10 . It is shown that the present FFT results confirm that the stress contour reported in Fig. 9 respectively, are similar to that of an edge dislocation (see for instance [46] and [51]). 


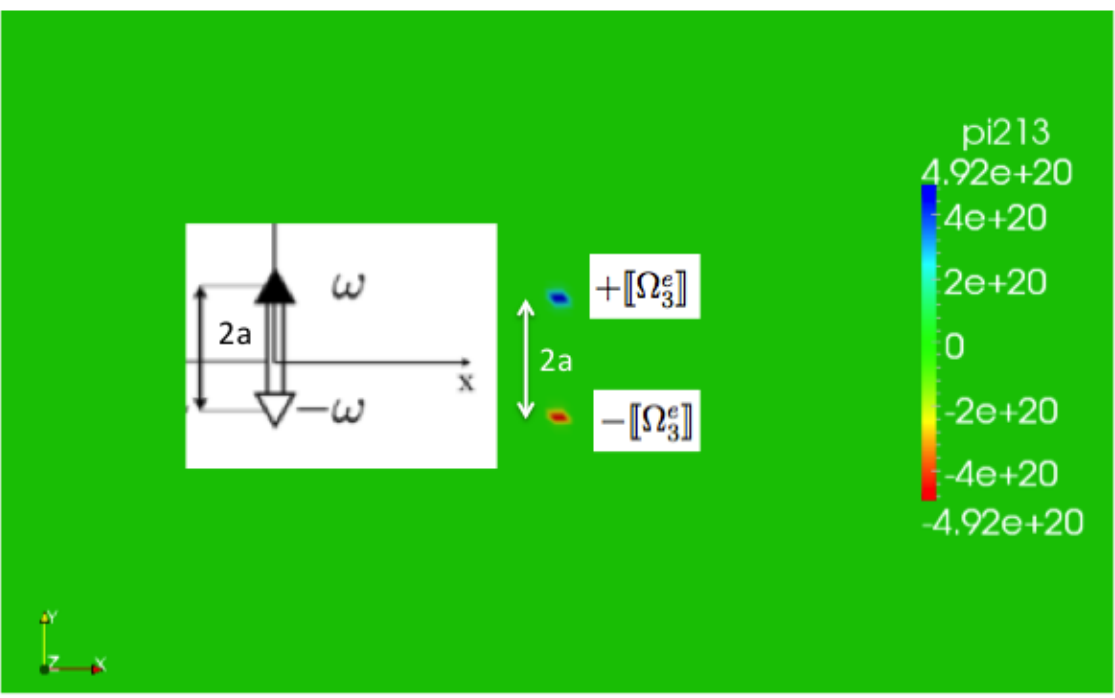

Fig. 5 Spatial distribution of G-disclination density in $\mathrm{rad} . \mathrm{m}^{-2}$ for a straight wedge disclination dipole with associated positive and negative rotation jumps: $+\llbracket \Omega_{3}^{e} \rrbracket$ and $-\llbracket \Omega_{3}^{e} \rrbracket$.

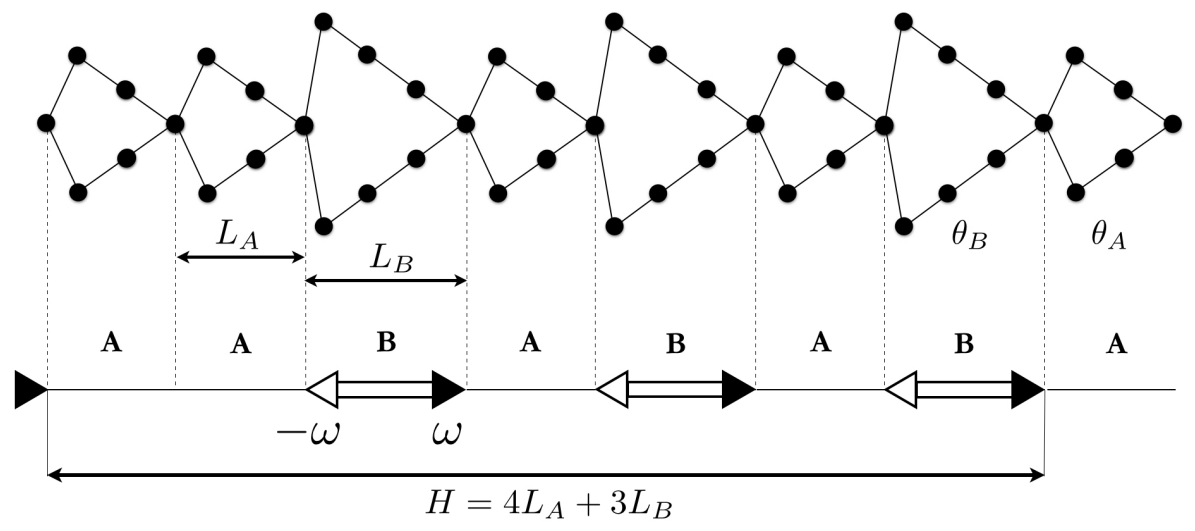

Fig. 6 Example of a disclination Structural Unit Model (DSUM) representation of the [001] $\Sigma 149(1070) \theta=20.02^{\circ}$ symmetric tilt grain boundary. Its structural decomposition is - AABABAB.AABABAB - , with B being the minority structural unit. The elastic fields of this grain boundary can be constructed as the superposition of three offsetted periodic walls of disclination dipoles B. 

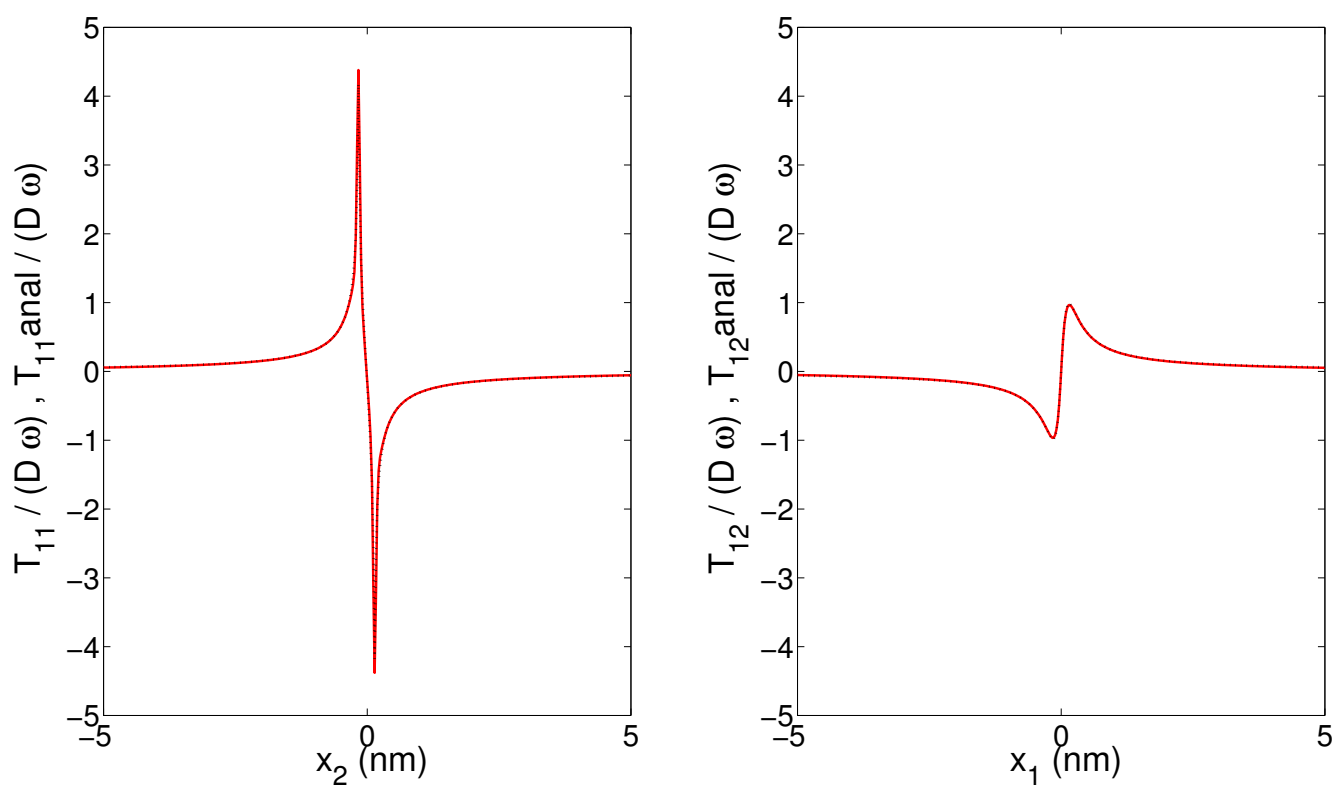

Fig. 7 Profiles of stress components: (a) $T_{11}$, (b) $T_{12}$, for a straight wedge disclination dipole (see Fig. 5) obtained by FFT and normalized by $D \omega$ where $D=\mu /(2 \pi(1-v))$ (solid lines). Comparisons with the solutions given by $[5,10]$ (dashed lines).
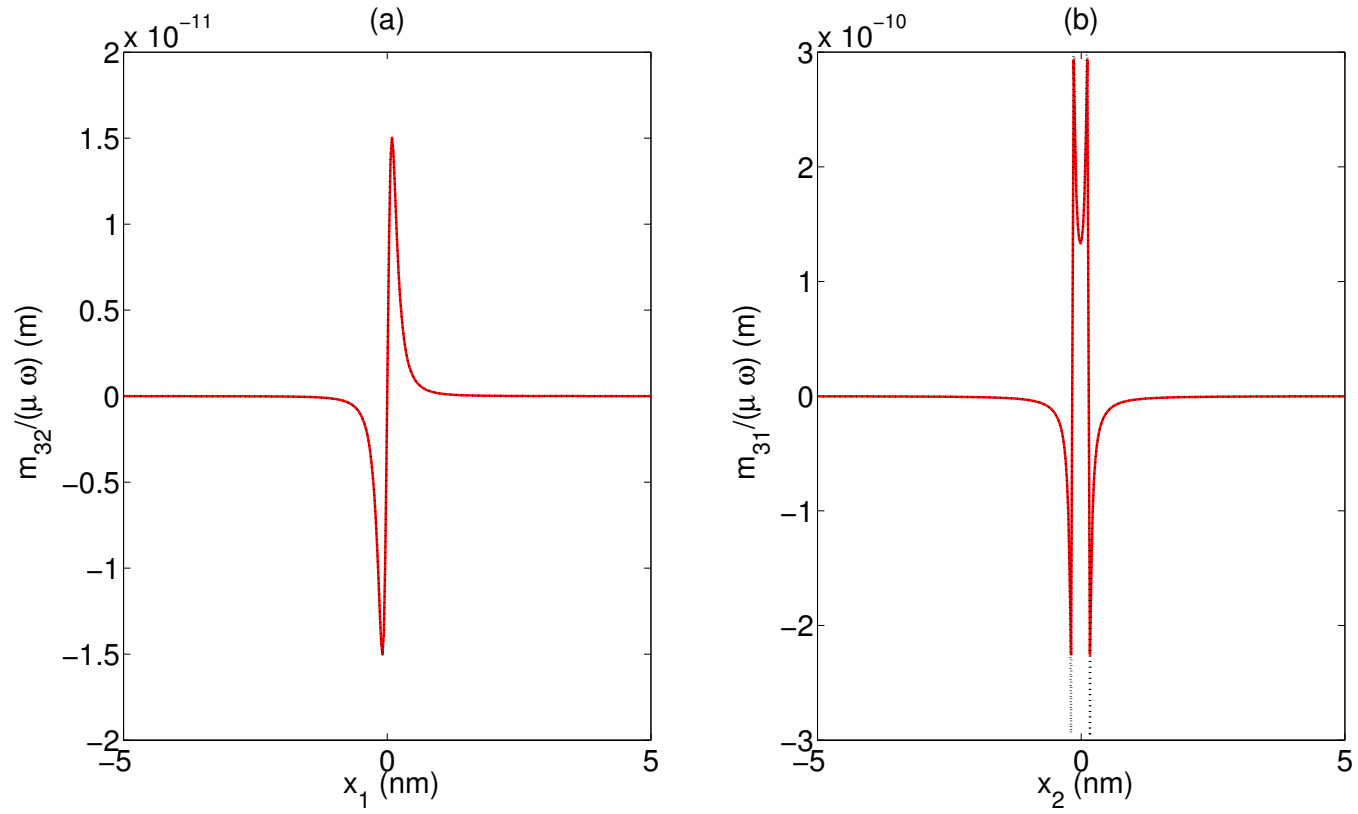

Fig. 8 Profiles of couple stress components: (a) $m_{32}^{D}$, (b) $m_{31}^{D}$, for a straight wedge disclination dipole (see Fig. 5) obtained by FFT and normalized by $\mu \omega$ (solid lines). Comparisons with the solutions given by $[5,10]$ (dashed lines). 


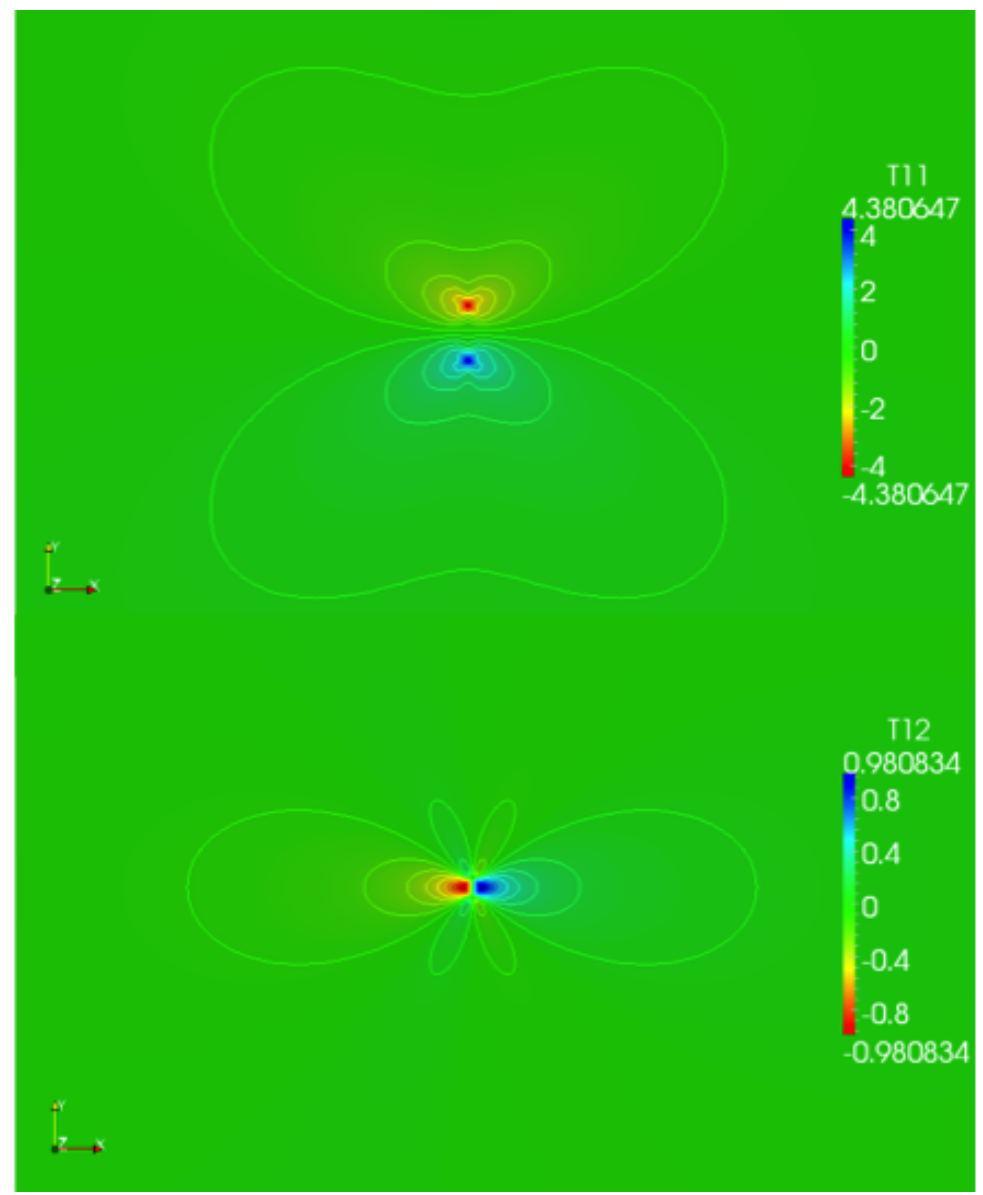

Fig. 9 Stress contours for $T_{11}$ (top) and $T_{12}$ (bottom) for a straight wedge disclination dipole (see Fig. 5) obtained by FFT (a,c) and normalized by $D \omega$ where $\omega=\llbracket \Omega_{3}^{e} \rrbracket$ and $D=\mu /(2 \pi(1-v))$. 


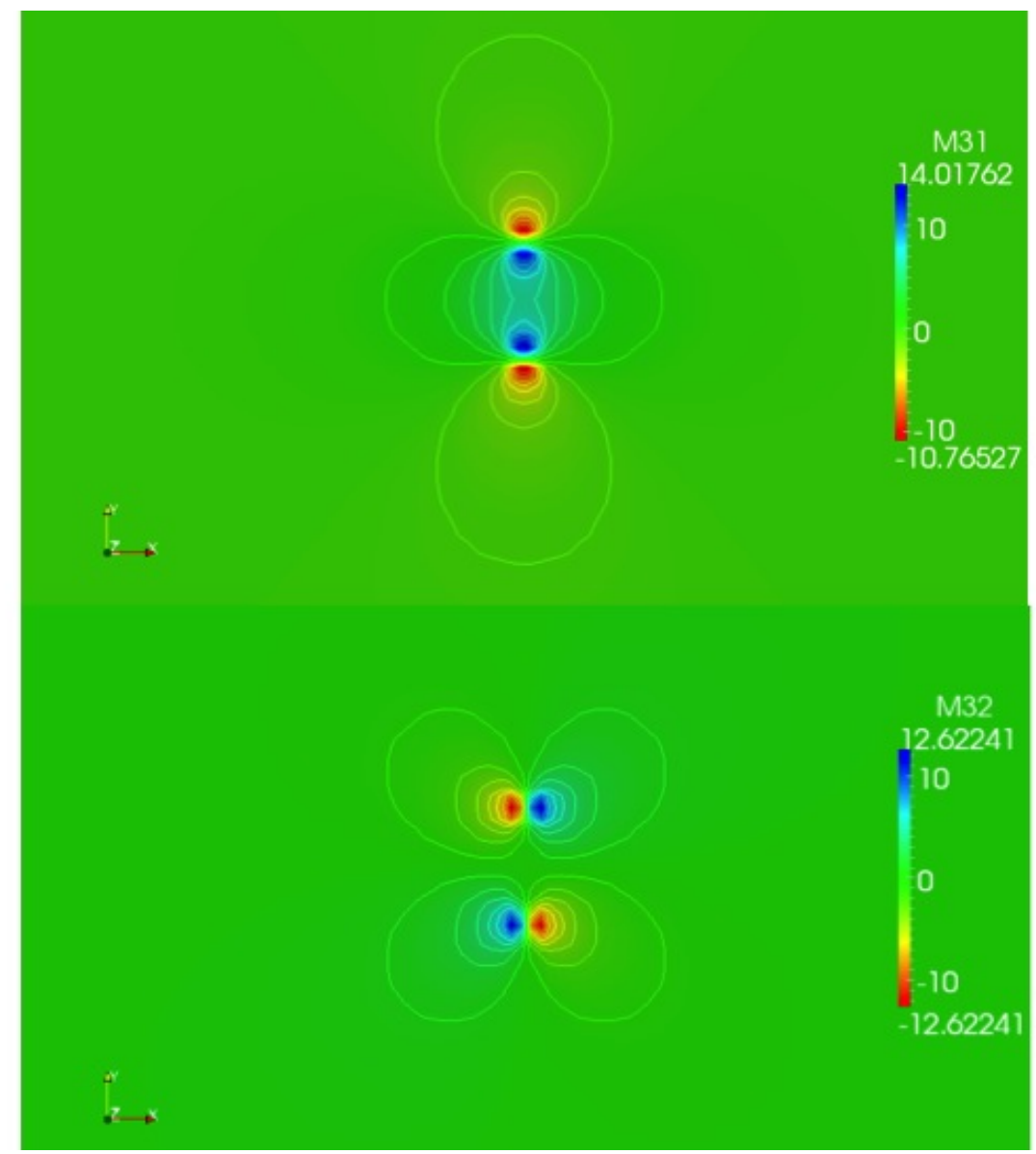

Fig. 10 Couple stress contours for $m_{31}^{D}$ (top) and $m_{32}^{D}$ (bottom) for a straight wedge disclination dipole (see Fig. 5) obtained by FFT $(\mathrm{a}, \mathrm{c})$ and normalized by $\omega$ where $\omega=\llbracket \Omega_{3}^{e} \rrbracket$.

\section{Concluding remarks}

A spectral approach for periodic media was developed to solve the elasto-static field equations of gdisclination mechanics [2,3] in the framework of a couple stress theory as an extension of the work described in [6], which was only dedicated to a Cauchy stress theory. Various results are obtained such as the solutions of Poisson-type equations in the Fourier space for a medium containing both dislocation and g-disclination densities. These solutions capture the incompatible part of elastic fields induced by gdisclinations in the Fourier space. The compatible elastic fields needed to retrieve the stress and couple stress fields are solved assuming a characteristic internal length scale $l$ involved in the equilibrium equations and associated with couple stresses. The latter is chosen to be related to the Burgers vector $(l=0.5 b)$ of the material to give realistic and physical elastic fields. The present discrete Fourier transform method uses the FFT algorithm and has been adapted to 2D periodic unit cells containing straight wedge disclinations in isotropic elasticity where defect line is parallel to the third dimension (i.e. $x_{3}$ axis). The discrete Fourier transform method is based on differentiation rules up to fourth-order partial derivatives allowing accurate calculations of stress and couple stress fields in comparison with analytical solutions for single disclinations or associated dipoles. The present theory and FFT implementation may be useful to derive the internal stress and couple stress fields of grain boundaries seen as DSUM (Disclination Structural Unit Model) [18] or more generalized defects which may include a combination of stretching and rotation-discontinuities at the nanoscale. Furthermore, the numerical framework presented in this paper can be easily adapted to elastic anisotropic materials (see eqs. 58 to 60 ). 
Acknowledgements SB would like to thank Professors P. Neff, W. H. Müller and S. Forest for fruitful discussions during the conference. This work is supported by the French State through the National Research Agency (ANR) under the program Investment in the future (LabEx DAMAS referenced as ANR-11-LABX-0008-01).

\section{References}

1. Acharya, A.: A model of crystal plasticity based on the theory of continuously distributed dislocations. J. Mech. Phys. Solids 49, 761-785 (2001)

2. Acharya, A., Fressengeas, C.: Coupled phase transformations and plasticity as a field theory of deformation incompatibility. Int. J. Fract. 174, 87-94 (2012)

3. Acharya, A., Fressengeas, C.: Continuum mechanics of the interaction of phase boundaries and dislocations in solids. In: Proceedings in Mathematics and Statistics for Workshop on Differential Geometry and Continuum Mechanics, Edinburgh, vol. 137, pp. 125-168. G. Q Chen, M. Grinfeld, R.J. Knops (eds.), Springer (2015)

4. Acharya, A., Roy, A.: Size effects and idealized dislocation microstructure at small scales: predictions of a Phenomenological model of Mesoscopic Field Dislocation Mechanics: Part I. J. Mech. Phys. Solids 54, 1687-1710 (2006)

5. Anthony, K.H.: Die Theorie der Disklinationen. Arch. Rat. Mech. Anal. 39, 43-88 (1970)

6. Berbenni, S., Taupin, V., Djaka, K.S., Fressengeas, C.: A numerical spectral approach for solving elasto-static field dislocation and g-disclination mechanics. Int. J. Solids Structures 51, 4157-4175 (2014)

7. Bilby, B.A., Bullough, R., Smith, E.: Continuous distributions of dislocations: a new application of the methods of nonRiemannian geometry. Proc. Roy. Soc. London A 231, 263-273 (1955)

8. Brenner, R., Beaudoin, A.J., Suquet, P., Acharya, A.: Numerical implementation of static field dislocation mechanics theory for periodic media. Philos. Mag. 94, 1764-1787 (2014)

9. deWit, R.: Linear theory of static disclinations. In: Fundamental aspects of dislocation theory, pp. 651-680. Simmons, J.A., de Wit, R., Bullough, R. NBS Spec. Publ. 317, vol. 1, (National Bureau of Standards, Washington, DC) (1970)

10. deWit, R.: Theory of disclinations: IV. Straight disclinations. J. Res. Nat. Bureau of Standards, A. Physics and Chemistry 77A(5), 607-658 (1973)

11. Dreyer, W., Müller, W.H., Olschewski, J.: An approximate analytical 2D-solution for the stresses and strains in eigenstrained cubic materials. Acta Mech. 136(3-4), 171-192 (1999)

12. Eringen, A.C.: Non local continuum field theories. Springer, New York (2002)

13. Eyre, D.J., Milton, G.W.: A fast numerical scheme for computing the response of composites using grid refinement. Eur. Phys. J. Appl. Phys. 6, 41-47 (1999)

14. Forest, S.: Milieux continus généralisés et matériaux hétérogènes. Presses de l’Ecole des Mines, Paris (2006)

15. Fressengeas, C., Taupin, V., Capolungo, L.: An elasto-plastic theory of dislocation and disclination fields. Int. J. Solids Structures 48, 3499-3509 (2011)

16. Fressengeas, C., Taupin, V., Capolungo, L.: Continuous modeling of the structure of symmetric tilt boundaries. Int. J. Solids Structures 51(6), 1434-1441 (2014)

17. Frigo, M., Johnson, S.G.: The design and implementation of FFTW3. Proceedings of the IEEE 93(2), 216-231 (2005)

18. Gertsman, V.Y., Nazarov, A.A., Romanov, A.E., Valiev, R.Z., Vladimirov, V.I.: Disclination-structural unit model of grain boundaries. Philos. Mag. A 59(5), 1113-1118 (1989)

19. Gourgiotis, P.A., Georgiadis, H.G.: An approach based on distributed dislocations and disclinations for crack problems in couple-stress theory. Int. J. Solids Structures 45, 5521-5539 (2008)

20. Hirth, J.P., Lothe, J.: Theory of Dislocations (2nd ed.). Wiley, New York (1982)

21. Jiang, B.: The least-squares finite element method, in: Theory and Computation in Fluid Dynamics and Electromagnetics. Springer Series in Scientific Computation, Springer, Berlin (1998)

22. Kassbohm, S.: Fourierreihen zur Berechnung repräsentativer Volumenelemente mit Mikrostruktur. Ph.D. thesis, Fakultät V der TU Berlin, Berlin, Germany (2006)

23. Kassbohm, S., Müller, W.H., Silber, G., Fessler, R.: Fourier Series for Continua with Microstructure. PAMM - Proc. Appl. Math. Mech. 6, 487-488 (2006)

24. Koiter, W.T.: Couple stresses in the theory of elasticity, I and II. Proceedings of the Koninklijke Nederlandse Akademie van Wetenschappen, Series B 67, 17-44 (1964)

25. Kosevich, A.M.: Crystal dislocations and the theory of elasticity (chap. 1). In: Dislocations in Solids (vol. 1), F.R.N. Nabarro, ed., pp. 33-141. Amsterdam, North-Holland (1979)

26. Kröner, E.: Kontinuumstheorie der Versetzungen und Eigenspannungen. Collatz L. and Loesch F. (eds.). Ergebnisse der Angewewandte Mathematik 5, Springer Verlag, Berlin (1958)

27. Kröner, E.: On the physical reality of torque stresses in continuum mechanics. Int. J. Engng. Sci. 1, 261-278 (1963)

28. Kröner, E.: Mechanics of Generalized Media. Proceedings of the IUTAM Symposium on the generalized Cosserat continuum and the continuum theory of dislocations with applications. Kröner, E. (ed.), Springer Verlag, Berlin (1968)

29. Kröner, E.: Continuum theory of defects. In: Physics of defects, pp. 215-315. R. Balian et al. (Eds.), Les Houches, Session 35, North Holland, New York (1981)

30. Lebensohn, R.A.: N-site modeling of a 3D viscoplastic polycrystal using Fast Fourier Transform. Acta Mater. 49, 2723$2737(2001)$

31. Li, J.C.M.: Disclination model of high angle grain boundaries. Surface Science 31, 12-26 (1972)

32. Lubarda, V.A.: The effects of couple stresses on dislocation strain energy. Int. J. Solids Structures 40, 3807-3826 (2003)

33. Michel, J.C., Moulinec, H., Suquet, P.: A computational scheme for linear and non-linear composites with arbitrary phase contrast. Int. J. Num. Methods Engrg. 52, 139-160 (2001) 
34. Mindlin, R.D., Tiersten, H.F.: Effects of couple-stresses in linear elasticity. Arch. Rat. Mech. Anal. 11, 415-488 (1962)

35. Moulinec, H., Suquet, P.: A fast numerical method for computing the linear and non linear properties of composites. C. R. Acad. Sci. Paris II 318, 1417-1423 (1994)

36. Moulinec, H., Suquet, P.: A numerical method for computing the overall response of nonlinear composites with complex microstructure. Comput. Methods Appl. Mech. Engrg. 157, 69-94 (1998)

37. Müller, W.H.: Mathematical vs. experimental stress analysis of inhomogeneities in solids. J. Phys. IV 6(C1), 139-148 (1996)

38. Mura, T.: Continuous distribution of moving dislocations. Philos. Mag. 89, 843-857 (1963)

39. Nazarov, A.A., Shenderova, O.A., Brenner, D.W.: On the disclination-structural unit model of grain boundaries. Mater. Sci. Eng. A 281(1), 148-155 (2000)

40. Neff, P., Jeong, J., Ramidreza, H.: Subgrid interaction and micro-randomness - Novel invariance requirements in infinitesimal gradient elasticity. Int. J. Solids Structures 46, 4261-4276 (2009)

41. Neumann, S., Herrmann, K.P., Müller, W.H.: Stress/strain computation in heterogeneous bodies with discrete fourier transforms - different approaches. Comp. Mater. Sci. 25, 151-158 (2002)

42. Nowacki, W.: Theory of asymmetric elasticity. Pergamon Press, Oxford (1986)

43. Nye, J.F.: Some geometrical relations in dislocated crystals. Acta Metall. 1, 153-162 (1953)

44. Prakash, A., Lebensohn, R.A.: Simulation of micro mechanical behavior of polycrystals: Finite Elements versus Fast Fourier Transforms. Modell. Simul. Mater. Sci. Eng. 17, 64,010-64,016 (2012)

45. Press, W.H., Teukolsky, S.A., Vetterling, W.T., Flannery, B.P.: Numerical recipes in C++. The art of scientific computing (2nd ed.). Cambridge University Press, USA (2002)

46. Romanov, A.E., Vladimirov, V.: Disclinations in crystalline solids (chap. 47). In: Dislocations in Solids (vol. 9), pp. 191-402. Elsevier Science Publishers B.V. (1992)

47. Roy, A., Acharya, A.: Finite element approximation of field dislocation mechanics. J. Mech. Phys. Solids 53, 143-170 (2005)

48. Smyshlyaev, V.P., Fleck, N.A.: Bounds and estimates for linear composites with strain gradient effects. J. Mech. Phys. Solids 42, 1851-1882 (1994)

49. Taupin, V., Capolungo, L., Fressengeas, C., Das, A., Upadhyay, M.: Grain boundary modeling using an elasto-plastic theory of dislocation and disclination fields. J. Mech. Phys. Solids 61, 370-384 (2013)

50. Upadhyay, M.V.: On the role of defect incompatibilities on mechanical properties of polycrystalline aggregates: a multiscale study. Ph.D. thesis, School of Mechanical Engineering, Georgia Institute of Technology, Altanta, USA (2014)

51. Upadhyay, M.V., Capolungo, L., Taupin, V., Fressengeas, C.: Grain boundary and triple junction energies in crystalline media: a disclination based approach. Int. J. Solids Structures 48(22), 3176-3193 (2011)

52. Upadhyay, M.V., Capolungo, L., Taupin, V., Fressengeas, C.: Elastic constitutive laws for incompatible crystalline media: the contributions of dislocations, disclinations and g-disclinations. Philos. Mag. 93, 794-832 (2013)

53. Vinogradov, V., Milton, G.W.: An accelerated FFT algorithm for thermoelastic and non-linear composites. Int. J. Num. Meth. Eng. 76, 1678-1695 (2008)

54. Volterra, S.: Sur l'équilibre des corps élastiques multiplement connexes. Ann. Sci. Ecol. Norm. Sup. III 24, 401-517 (1907)

55. Willis, J.R.: Second-order effects of dislocations in anisotropic crystals. Int. J. Eng. Sci. 5, 171-190 (1967)

56. Zheng, Q.S., Zhao, Z.: Green's function and Eshelby's fields in couple-stress elasticity. Int. J. Multiscale Comput. Eng. 2, 15-27 (2004) 